\title{
Kampo formulas alleviate aging-related emotional disturbances and neuroinflammation in male senescence-accelerated mouse prone 8 mice
}

\author{
Naoki Ito ${ }^{1, *}$, Akiko Maruko, ${ }^{2}$, Kenshiro Oshima², Masaaki Yoshida ${ }^{3}$, Kengo Honma ${ }^{4}$, Chika \\ Sugiyama ${ }^{5}$, Takayuki Nagai ${ }^{1,4,6}$, Yoshinori Kobayashi ${ }^{1,7}$, Hiroshi Odaguchi ${ }^{1,5}$, Norihiro Okada ${ }^{2}$ \\ ${ }^{1}$ Oriental Medicine Research Center, Kitasato University, Tokyo 108-8642, Japan \\ ${ }^{2}$ Laboratory of Genomics for Health and Longevity, School of Pharmacy, Kitasato University, Sagamihara, \\ Kanagawa 252-0373, Japan \\ ${ }^{3}$ Research Laboratory, Kotaro Pharmaceutical Co., Ltd., Hakusan, Ishikawa 920-0201, Japan \\ ${ }^{4}$ Graduate School of Infection Control Sciences, Kitasato University, Tokyo 108-8642, Japan \\ ${ }^{5}$ Graduate School of Medical Sciences, Kitasato University, Sagamihara, Kanagawa 252-0373, Japan \\ ${ }^{6}$ Laboratory of Biochemical Pharmacology for Phytomedicines, Ōmura Satoshi Memorial Institute, Kitasato \\ University, Tokyo 108-8642, Japan \\ ${ }^{7}$ Department of Pharmacognosy, School of Pharmacy, Kitasato University, Minato-ku, Tokyo 108-8642, Japan \\ *Equal contribution
}

Correspondence to: Naoki Ito; email: ito-n@insti.kitasato-u.ac.jp

Keywords: aging, emotional disturbances, kososan, neuroinflammation, SAMP8 mice

Received: October 13, $2021 \quad$ Accepted: December 20, $2021 \quad$ Published: January 3, 2022

Copyright: (C) 2022 Ito et al. This is an open access article distributed under the terms of the Creative Commons Attribution License (CC BY 3.0), which permits unrestricted use, distribution, and reproduction in any medium, provided the original author and source are credited.

\begin{abstract}
Aging-induced neuroinflammation, also known as neuroinflammaging, plays a pivotal role in emotional disturbances, including depression and anxiety, in older individuals, thereby leading to cognitive dysfunction. Although numerous studies have focused on therapeutic strategies for cognitive impairment in older individuals, little research has been performed on treating its preceding emotional disturbances. Here, we examined whether Kampo formulas (kososan [KS], nobiletin-rich kososan [NKS], and hachimijiogan [HJG]) can ameliorate aging-induced emotional disturbances and neuroinflammation in mice. The depression-like behaviors observed in SAMP8 mice, relative to normally aging SAMR1 mice, were significantly prevented by treatment with Kampo formulas for 13 weeks. Western blot analysis revealed that hippocampal neuroinflammation was significantly abrogated by Kampo formulas. KS and NKS also significantly attenuated the hippocampal neuroinflammatory priming induced by lipopolysaccharide (LPS, $0.33 \mathrm{mg} / \mathrm{kg}$, i.p.) challenge in SAMP8 mice. Hippocampal IL-1 $\beta$, IL-6, and MCP-1 levels were significantly decreased in NKS-treated SAMP8 mice. KS and NKS showed significantly reduced tau accumulation in the brains of SAMP8 mice. RNA-sequencing revealed that each Kampo formula led to unique dynamics of hippocampal gene expression and appeared to abrogate hippocampal inflammatory responses. HJG significantly blocked the LPS-induced increase in serum IL6 and MCP-1. These results suggest that Kampo formulas would be useful for treating aging-induced depression, in part by regulating neuroinflammatory pathways. This finding may pave the way for the development of therapeutic strategies for aging-related emotional disturbances, which may contribute to the prevention of cognitive dysfunction in older individuals.
\end{abstract}




\section{INTRODUCTION}

Aging is a risk factor for various diseases, including emotional disorders $[1,2]$, neurodegenerative diseases [3], anorexia [4], diabetes [5], cardiovascular diseases [6], osteoporosis [7], presbyopia [8], hypoacusis [9], and sarcopenia [10]. Most of these diseases are linked to chronic inflammation associated with aging [11]. Numerous studies have demonstrated that neurodegenerative diseases are strongly associated with neuroinflammation, which is aggravated by aging (termed neuroinflammaging [12]) [13-15].

Recent studies have also reported that neuroinflammation plays an important role in the pathology of emotional disorders, including depression [16-20]. This suggests a close link between neuroinflammaging and depression, as demonstrated by our recent finding that neuroinflammaging underlies emotional disturbances in a senescence-accelerated mouse model [21]. Several animal and human studies have reported that existing antidepressants attenuate neuroinflammation [22-25], but because antidepressants affect the metabolism of glucose [26], lipids [27], and other drugs [28-31] and may lead to exacerbation of diseases comorbid with depression, attention should be paid to long-term antidepressant treatment in older individuals with polypharmacy. Moreover, a recent finding that showed that depression causes cognitive dysfunction deterioration in older individuals with small vessel disease [32] suggests that the onset of cognitive impairment could be accelerated by depressive pathology [33]. These findings underscore the need for treating depression in older individuals. It is thus desirable to establish safer and more-effective therapeutic strategies and develop alternative therapies for late-life depression.

Although antidepressants are available as general medication for depression, some Kampo formulas, which are traditional Japanese herbal medicines, have clinically beneficial effects on emotional disorders, including depressive mood and anxiety, in patients with widely varying age ranges. Among these, kososan (KS), which consists of five herbs (Cyperus rhizome, Perilla herb, Citrus unshiu peel, Glycyrrhiza, and Ginger), is currently used to treat depressive states as well as the common cold, allergic urticaria caused by ingested food, irritable bowel syndrome, chronic fatigue syndrome, insomnia, and autonomic imbalance. Our previous animal studies using experimental stress models have demonstrated that $\mathrm{KS}$ exerts antidepressant-like effects by normalizing the dysfunction of the hypothalamic-pituitary-adrenal axis [34, 35], regulating the orexin $\mathrm{A} /$ neuropeptide $\mathrm{Y}$ signaling system [36, 37], and modulating metabotropic glutamate receptor 2 and 2',3'-cyclic nucleotide 3'-phosphodiesterase 1 in the hypothalamus, according to a proteomic analysis [38]. Our recent study also found that KS prevented social avoidant behavior, a depressive-like behavior, presumably by attenuating hippocampal neuroinflammation in a social-defeat mouse model of depression [39]. Accumulating evidence has revealed that nobiletin, a polymethoxyflavone present in Citrus unshiu peel, has anti-inflammatory [40, 41], neuroprotective [42], and antidepressant-like activities [40, 43-45] and improves cognitive impairment [41, $46,47]$ in rodent studies. In addition, Citrus reticulata peel, which has a nobiletin content that is $\sim 16$-fold higher than that of Citrus unshiu peel, facilitates cAMP/PKA/ERK/CREB signaling in cultured hippocampal neurons [48] and may prevent the progression of cognitive dysfunction in patients with Alzheimer's disease [49]. Although no evidence has been reported on the effects of Citrus reticulata peel on emotional disturbances and neuroinflammation, it would be predicted that KS containing Citrus reticulata peel (nobiletin-rich $\mathrm{KS}$ [NKS]) is more effective for treating aging-induced emotional disturbances and neuroinflammation. Given these findings and assumptions, we hypothesized that KS would ameliorate depressive-like behaviors and neuroinflammation associated with aging and that the effects of NKS would be superior to those of KS.

To evaluate this hypothesis, we examined whether KS and NKS exerted antidepressant-like effects and neuroinflammation-suppressive effects in senescenceaccelerated mouse prone 8 (SAMP8) mice, which show depressive- and anxiety-like behaviors, circadian rhythm disruption, and neuroinflammation associated with aging [21]. Furthermore, we compared the effects of KS and NKS with those of another Kampo formula, hachimijiogan (HJG), which has traditionally been used to treat several aging-related disorders, including cognitive impairments $[50,51]$ and irritable bladder [52].

\section{RESULTS}

\section{Kampo formulas ameliorate aging-induced depression-like behaviors in SAMP8 mice}

In the TST, the immobility time of SAMP8 mice was significantly increased relative to that of senescenceaccelerated mouse resistant 1 (SAMR1) mice, as normal aging controls (Figure 1A; Bonferroni's post-hoc test, $P<0.0001)$. This effect was significantly prevented by treatment with Kampo formulas (Bonferroni's post-hoc test; KS, $P<0.0001$; NKS, $P<0.0001$; HJG, $P<0.001$ ). Moreover, a significant difference in immobility was 
found between NKS and HJG treatment (Bonferroni's post-hoc test, $P<0.001$ ), suggesting that the preventive effect of NKS was superior to that of HJG.

In the SPT, the sucrose preference was significantly lower in SAMP8 mice than in SAMR1 mice (Figure

A

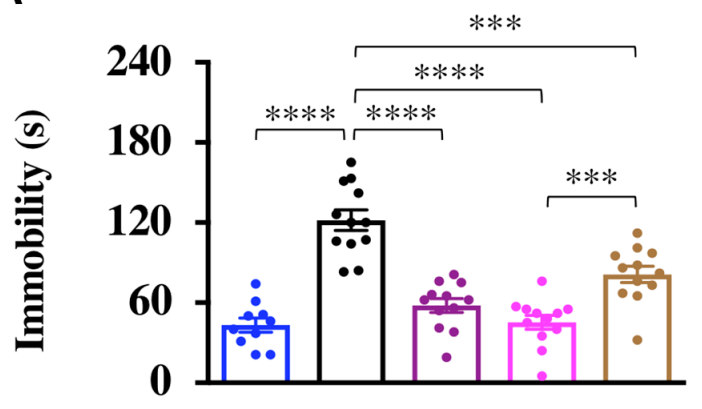

C

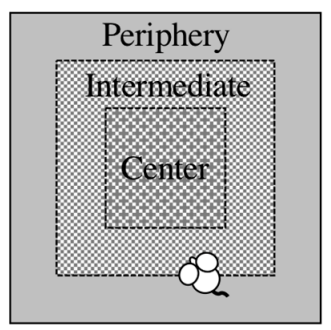

Center zone

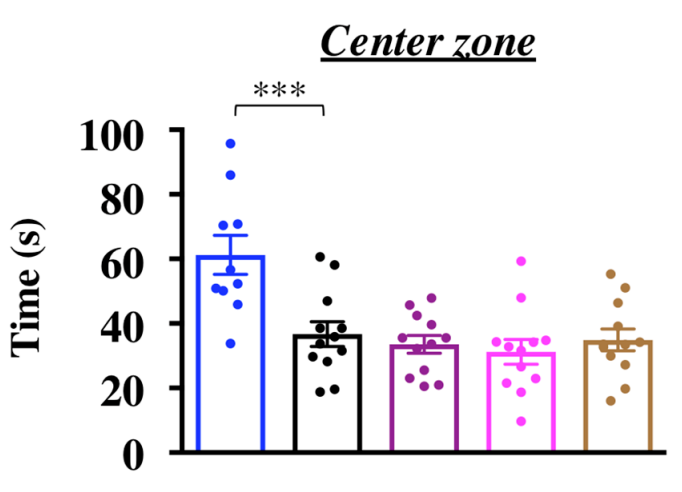

Peripheral zone

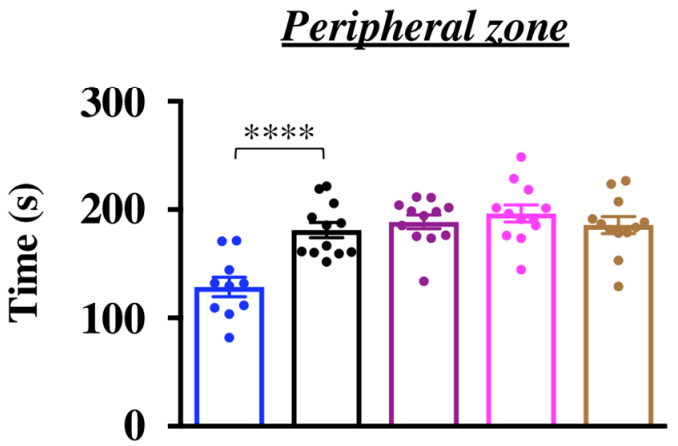

1B; Bonferroni's post-hoc test, $P<0.05$ ). This decrease in sucrose preference was not affected by treatment with Kampo formulas. However, a significant negative correlation was also found between the immobility time in the TST and the sucrose preference in the SPT (Supplementary Figure 1; Pearson's correlation

B

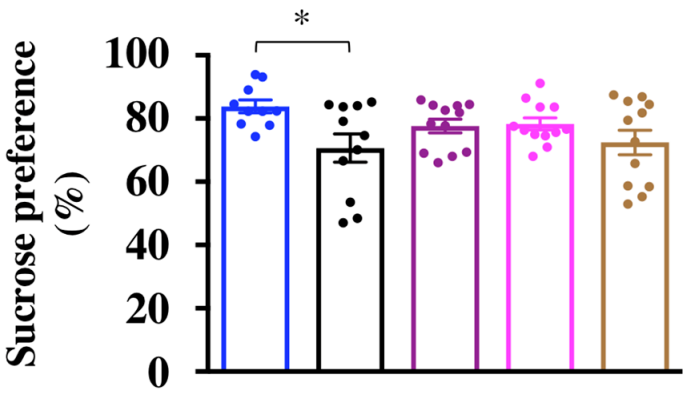

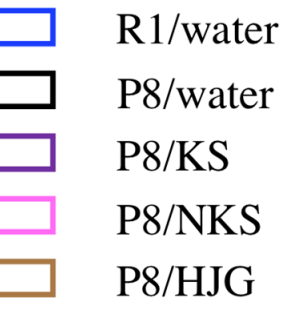

Intermediate zone

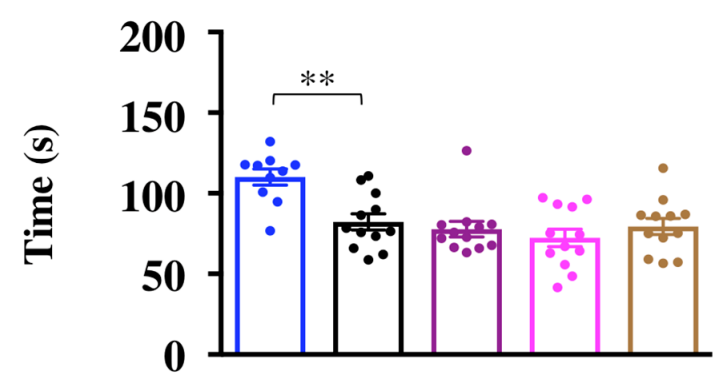

Distance traveled

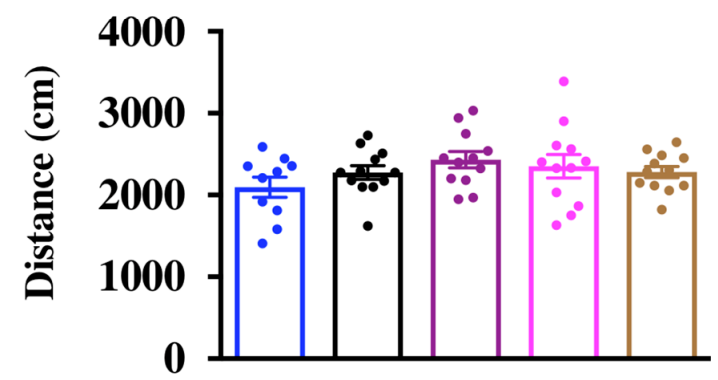

Figure 1. Oral administration of Kampo formulas ameliorates depression-like behaviors but not anxiety-like behaviors in SAMP8 mice. The tail suspension test (TST, A), sucrose preference test (SPT, B), and open field test (OFT, C) were performed following treatment with the indicated Kampo formulas or with water for 13 weeks. Data are shown as the mean \pm SEM $(n=10-12) .{ }^{*} P<0.05,{ }^{* *} P<0.01$, ${ }^{* * *} P<0.001$, and ${ }^{* * * *} P<0.0001$ using Bonferroni's post-hoc test. Abbreviations: KS: kososan; NKS: nobiletin-rich kososan; HJG: hachimijiogan. 
coefficient test, $r=-0.3048, P<0.05)$, suggesting that Kampo formulas ameliorate aging-induced apathy-like behavior, along with anhedonia-like behavior, in SAMP8 mice. This behavioral recovery indicates the antidepressant-like effect of the Kampo formulas.

In the OFT, the time spent in the center and intermediate zones was significantly decreased and the time spent in the peripheral zone was significantly increased in SAMP8 mice relative to SAMR1 mice (Figure 1C; Bonferroni's post-hoc test; center zone, $P<0.001$; intermediate zone, $P<0.01$; peripheral zone, $P<0.0001)$. These changes were retained after treatment with Kampo formulas. There was also no difference among the groups in the distance traveled during the OFT. Thus the Kampo formulas do not prevent aging-induced anxiety-like behaviors in SAMP8 mice.

At the beginning of the Kampo treatment period (7 weeks of age), mice in all groups had increased locomotor activity during the nocturnal phase relative to the diurnal phase (Figure 2A). Similarly, cumulative activity during the nocturnal phase was significantly higher than that during the diurnal phase for all groups (Figure 2C; paired $t$-test; SAMR1/water, $P<0.0001$; SAMP8/water, $P<0.05$; SAMP8/KS, $P<0.0001$; SAMP8/NKS, $P<0.001$; SAMP8/HJG, $P<0.001$ ), indicating that both SAMR1 and SAMP8 mice showed normal circadian rhythms at 7 weeks of age. At 19 weeks of age, however, there was no difference in cumulative activity between diurnal and nocturnal phases in the water-administered SAMP8 mice (Figure 2B, 2D). There was still a significant difference between these phases in the water-administered SAMR1 mice (paired $t$-test, $P<0.05$ ), although a nanotag datum of a water-administered SAMR1 mouse was not extracted because of the built-in battery exhaustion of nanotag. These results suggest that SAMP8 mice aged 19 weeks exhibit circadian rhythm disruption. This disruption was not alleviated by treatment with Kampo formulas, even when the SAMP8 mice were assessed at earlier ages (Supplementary Figure 2), implying that Kampo formulas are ineffective for treating circadian rhythm disruption in SAMP8 mice.

With regard to body weight changes, the wateradministered SAMP8 mice had significantly greater body weight gain from 7 to 19 weeks of age as compared with the water-administered SAMR1 mice (Bonferroni's post-hoc test, $P<0.05$ ). No differences in body weight were found between water-administered and Kampo formulas-administered SAMP8 mice (Supplementary Figure 3).

\section{Hippocampal neuroinflammatory priming is attenuated in different ways by Kampo formulas in SAMP8 mice}

To unravel the mechanisms underlying the antidepressant-like effect of Kampo formulas, we examined whether Kampo formulas regulate neuroinflammation in the hippocampus, which is involved in the depressive phenotype [53-55]. We used expression of NLRP3 and Arg 1 as markers of pro- and anti-inflammatory phenotypes, respectively. Under physiological conditions, there was no difference in NLRP3 expression among the groups (Figure 3A and $3 \mathrm{~B}$, left), whereas Arg1 expression tended to decrease in the water-administered SAMP8 mice relative to the water-administered SAMR1 mice (Figure $3 \mathrm{~A}$ and $3 \mathrm{C}$, left; Bonferroni's post-hoc test, $P<0.1)$. The decrease in Arg1 expression was significantly increased by treatment with Kampo formulas (Bonferroni's post-hoc test, $P<0.01)$. The ratio of NLRP3 to Arg1, which represents the balance between pro- and antiinflammatory phenotypes [21], was significantly increased in the water-administered SAMP8 mice as compared with the water-administered SAMR1 mice (Figure 3D, left; Bonferroni's post-hoc test, $P<0.05$ ). This increase was significantly reduced in SAMP8 mice treated with Kampo formulas (Bonferroni's post-hoc test, $P<0.01$ ), suggesting that Kampo formulas abrogate hippocampal neuroinflammation in SAMP8 mice aged 19 weeks.

Next, we examined whether Kampo formulas prevented the neuroinflammatory response to LPS injection, to assess the influence of neuroinflammatory priming on the hippocampus. NLRP3 expression was significantly increased in the water-administered SAMP8 mice as compared with the water-administered SAMR1 mice (Figure 3A and 3B, right; Bonferroni's post-hoc test, $P<$ 0.01 ), but this increase was not decreased by treatment with Kampo formulas. In contrast, Arg1 expression was significantly reduced in the water-administered SAMP8 mice as compared with the water-administered SAMR1 mice (Figure 3A and 3C, right; Bonferroni's post-hoc test, $P<0.0001)$, and the Arg1 expression was significantly increased in the NKS-administered SAMP8 mice relative to the water-administered SAMP8 mice (Bonferroni's post-hoc test, $P<0.05$ ). The ratio of NLRP3 to Arg1 was significantly increased in the wateradministered SAMP8 mice as compared with the wateradministered SAMR1 mice (Figure 3D, right; Bonferroni's post-hoc test, $P<0.0001)$. This increase in the NLRP3/Arg1 ratio was significantly decreased in KSand NKS-administered SAMP8 mice (Bonferroni's posthoc test; KS, $P<0.05$; NKS, $P<0.0001)$. These results suggest that hippocampal neuroinflammatory priming by LPS is buffered by treatment with KS and NKS. 
The expression of Toll-like receptor 4 (TLR4), an endogenous receptor for LPS, was also unaltered in all groups, irrespective of whether LPS had been administered (Supplementary Figure 4). This suggests that the inhibitory effects of KS and NKS on hippocampal neuroinflammatory priming do not result from the downregulation of TLR4 protein.

A
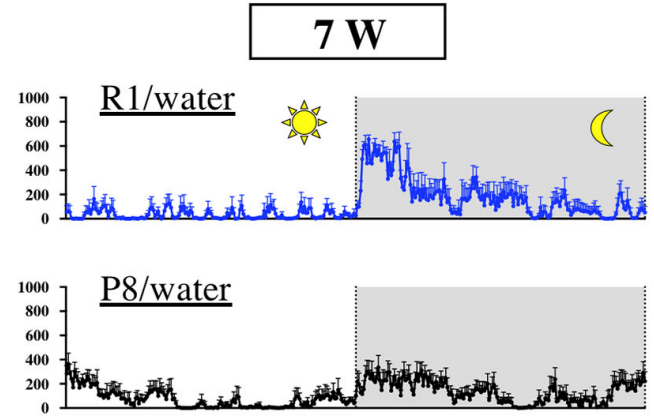

莺
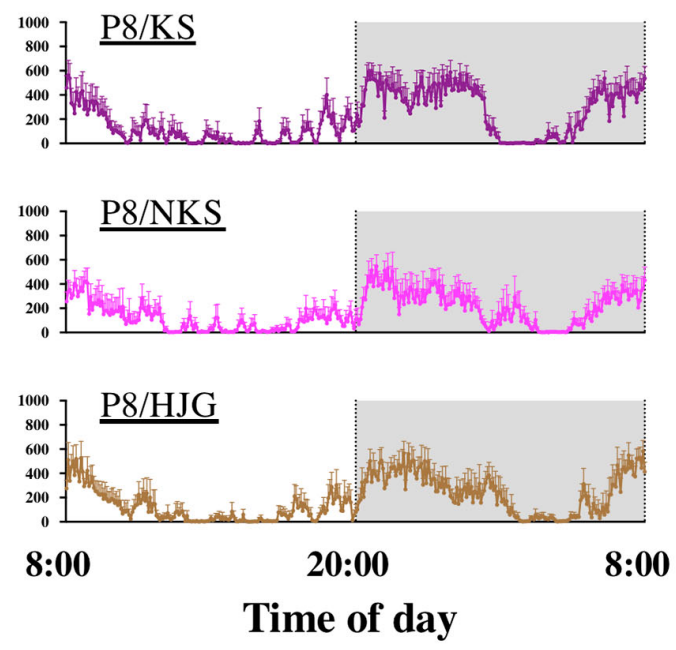

C

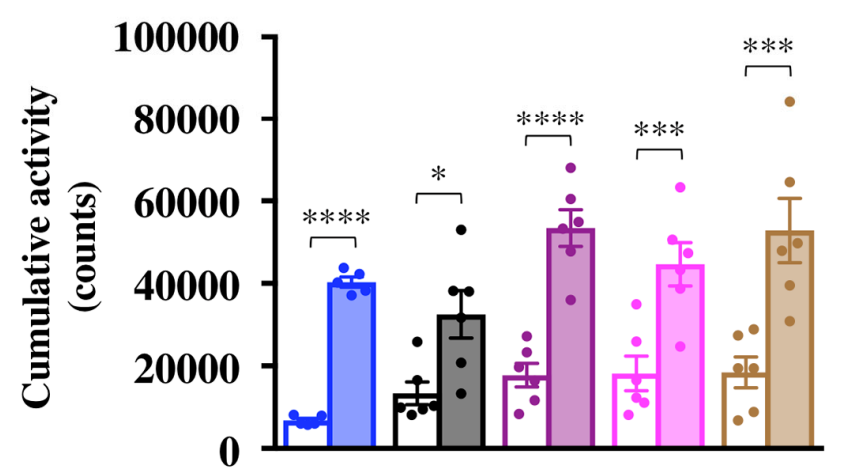

Kampo formulas result in characteristic gene expression patterns in the hippocampus of SAMP8 mice

To investigate whether Kampo formulas have characteristic effects on hippocampal gene expression dynamics, a genome-wide RNA-seq analysis was

\section{B}
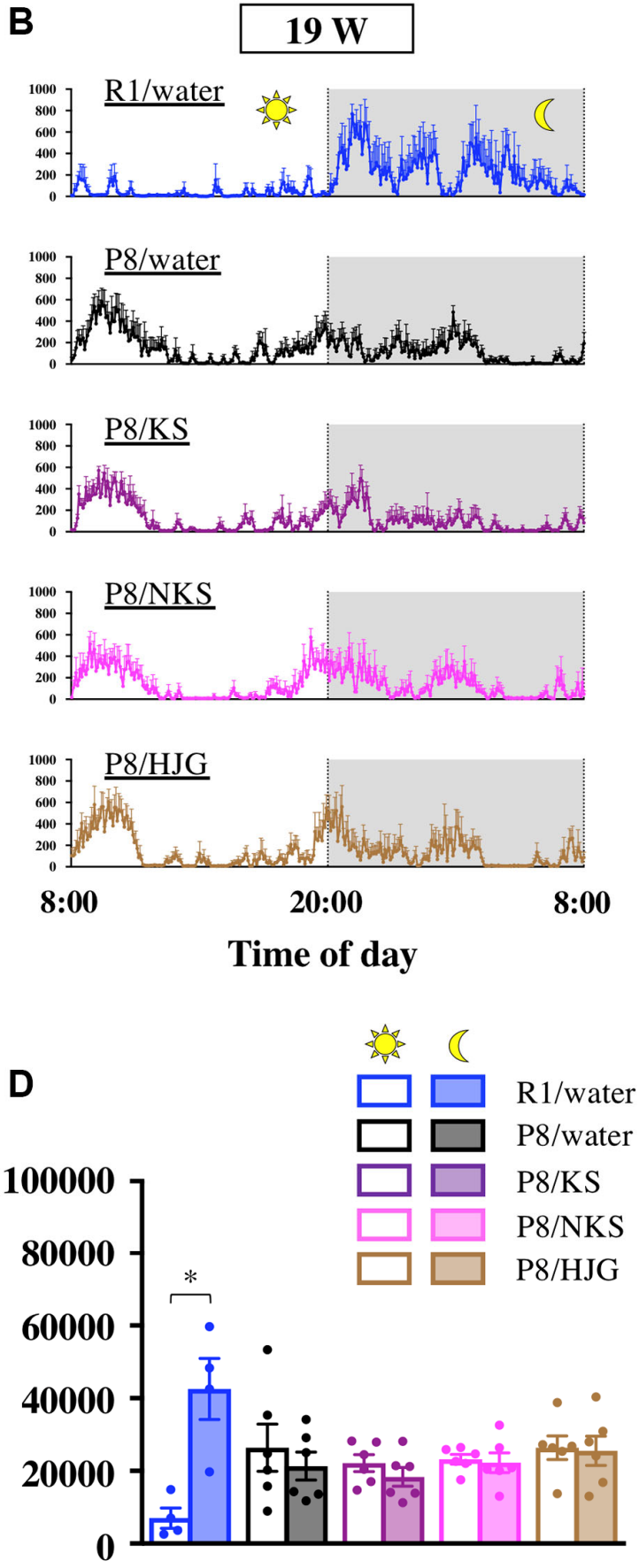

Figure 2. Oral administration of Kampo formulas does not improve circadian rhythm disruption in SAMP8 mice. Time course of locomotor activity of mice aged 7 weeks (A) and 19 weeks (B) in their home cages. The time between 8:00 and 20:00 and between 20:00 and 8:00 represents the diurnal and nocturnal phases, respectively. The cumulative activity during the diurnal and nocturnal phases of mice aged 7 weeks (C) and 19 weeks (D) is shown. Data are shown as the mean \pm SEM $(n=4-6) .{ }^{*} P<0.05,{ }^{* *} P<0.01,{ }^{* * *} P<0.001$, and ${ }^{* * * *} P<$ 0.0001 using paired $t$-test. Abbreviations: KS: kososan; NKS: nobiletin-rich kososan; HJG: hachimijiogan; W: weeks of age. 
conducted on hippocampal tissue from the treated mice. Genes that were significantly downregulated in the water-administered SAMP8 mice as compared with the water-administered SAMR1 mice and that were significantly upregulated by treatment with Kampo formulas were regarded as "V-shaped recovered genes."
Genes that were significantly upregulated in the wateradministered SAMP8 mice as compared with the wateradministered SAMR1 mice and that were significantly downregulated by treatment with Kampo formulas were regarded as "reverse $\mathrm{V}$-shaped recovered genes." On the basis of these criteria (Figure 4A), the V-shaped and
A

NLRP3

Arg1

GAPDH
Saline
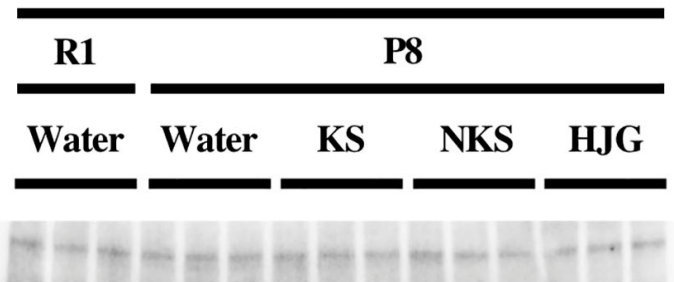

$-\infty-1-1-\infty-\infty$
LPS

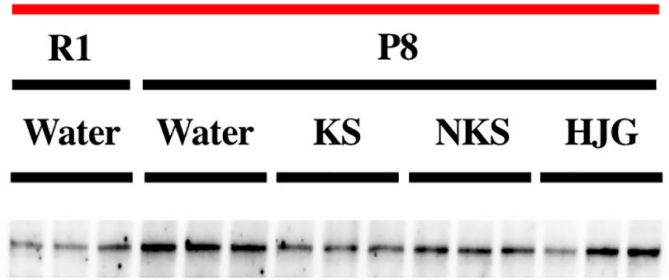

B

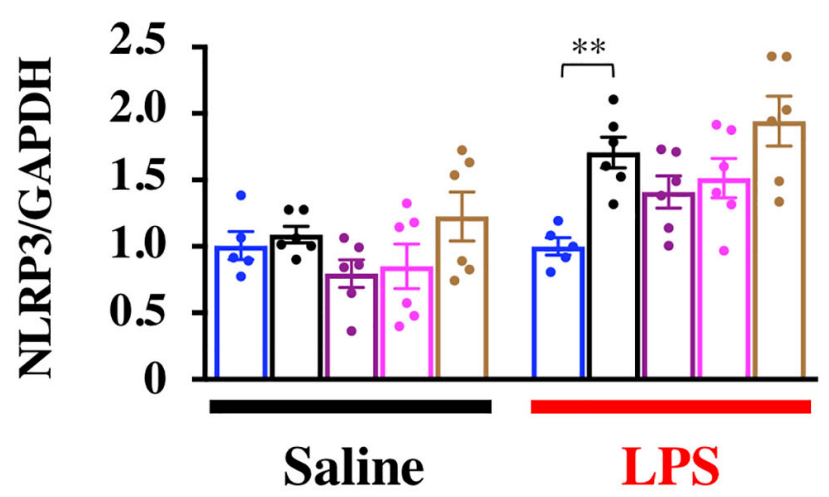

$\square \mathrm{R} 1 /$ water

$\square$ P8/water

$\square \mathrm{P} 8 / \mathrm{KS}$

$\square$ P8/NKS

$\square$ P8/HJG
C

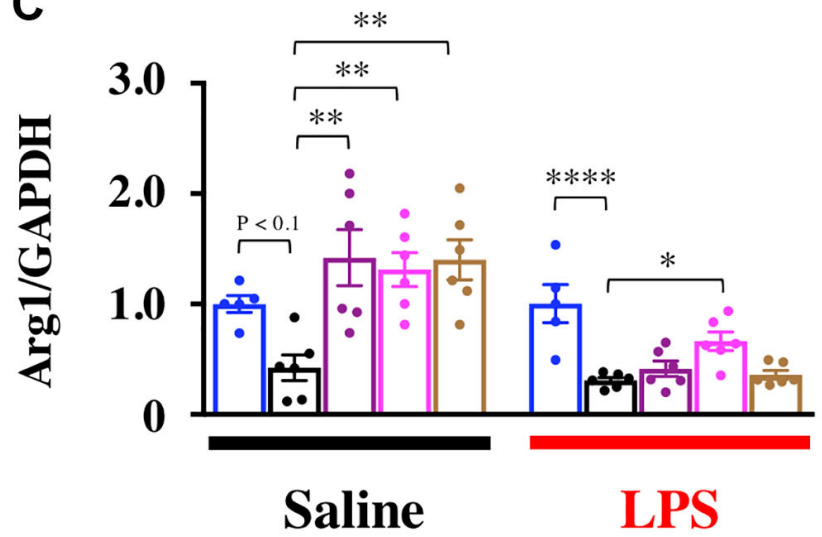

D

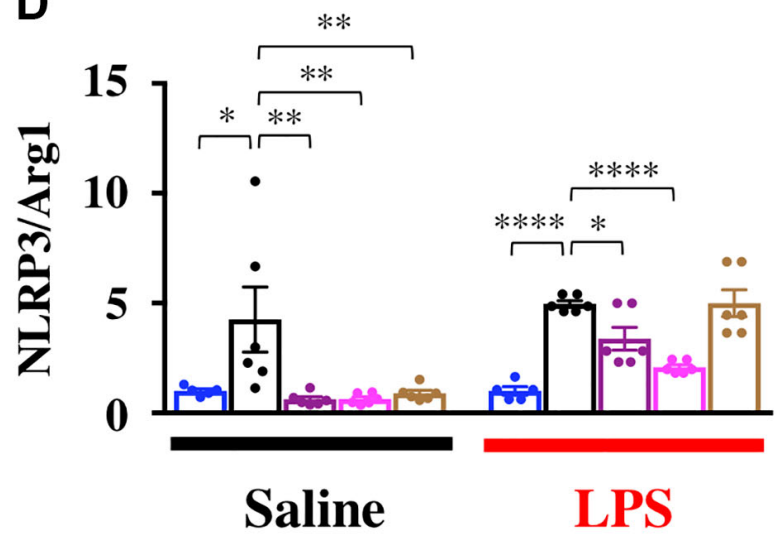

Figure 3. Distinct efficacy of different Kampo formulas for blocking neuroinflammatory priming in the hippocampus of SAMP8 mice at 19 weeks of age. (A) Representative western blotting images of NLRP3, Arg1, and GAPDH expression. Expression of NLRP3 (B) and Arg1 (C) were normalized based on GAPDH expression. (D) Pro-inflammatory/anti-inflammatory balance. Data are shown as the mean \pm SEM ( $n=5$ or 6 ). ${ }^{*} P<0.05,{ }^{* *} P<0.01,{ }^{* * *} P<0.001$, and ${ }^{* * * *} P<0.0001$ using Bonferroni's post-hoc test. Abbreviations: KS: kososan; NKS: nobiletin-rich kososan; HJG: hachimijiogan; LPS: lipopolysaccharide. 
reverse V-shaped recovered genes under physiological and LPS-stimulated conditions are summarized (Figure
4B), and a full list of significantly regulated genes is shown in Supplementary Table 1. The Venn diagrams
A

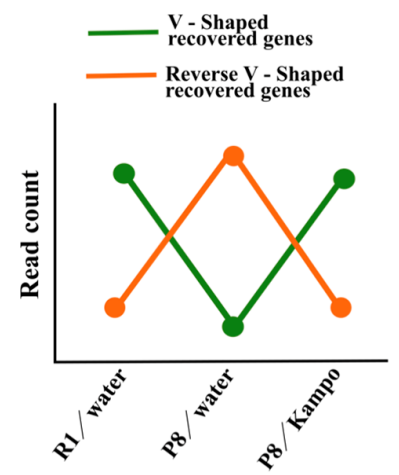

B

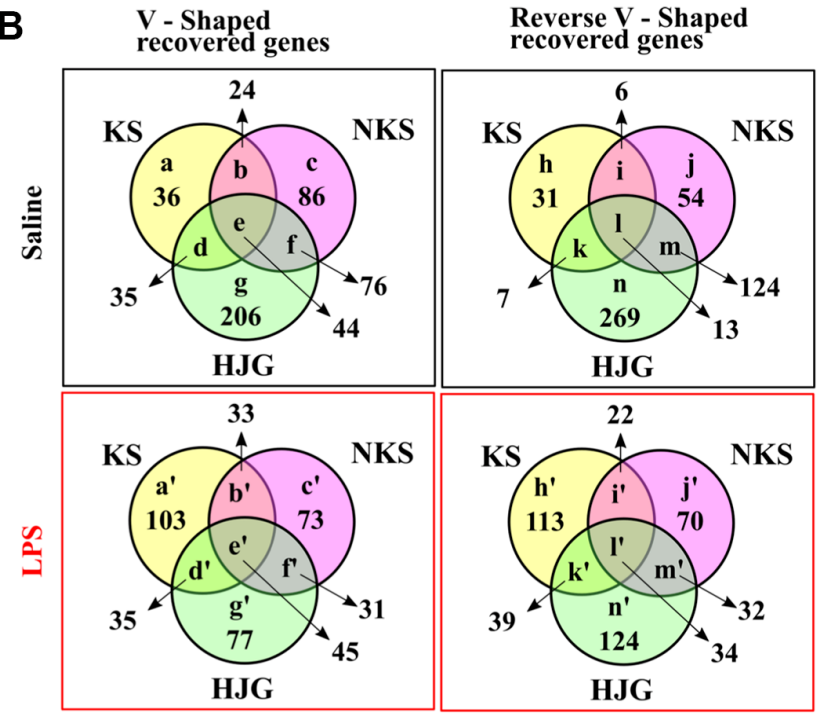

C
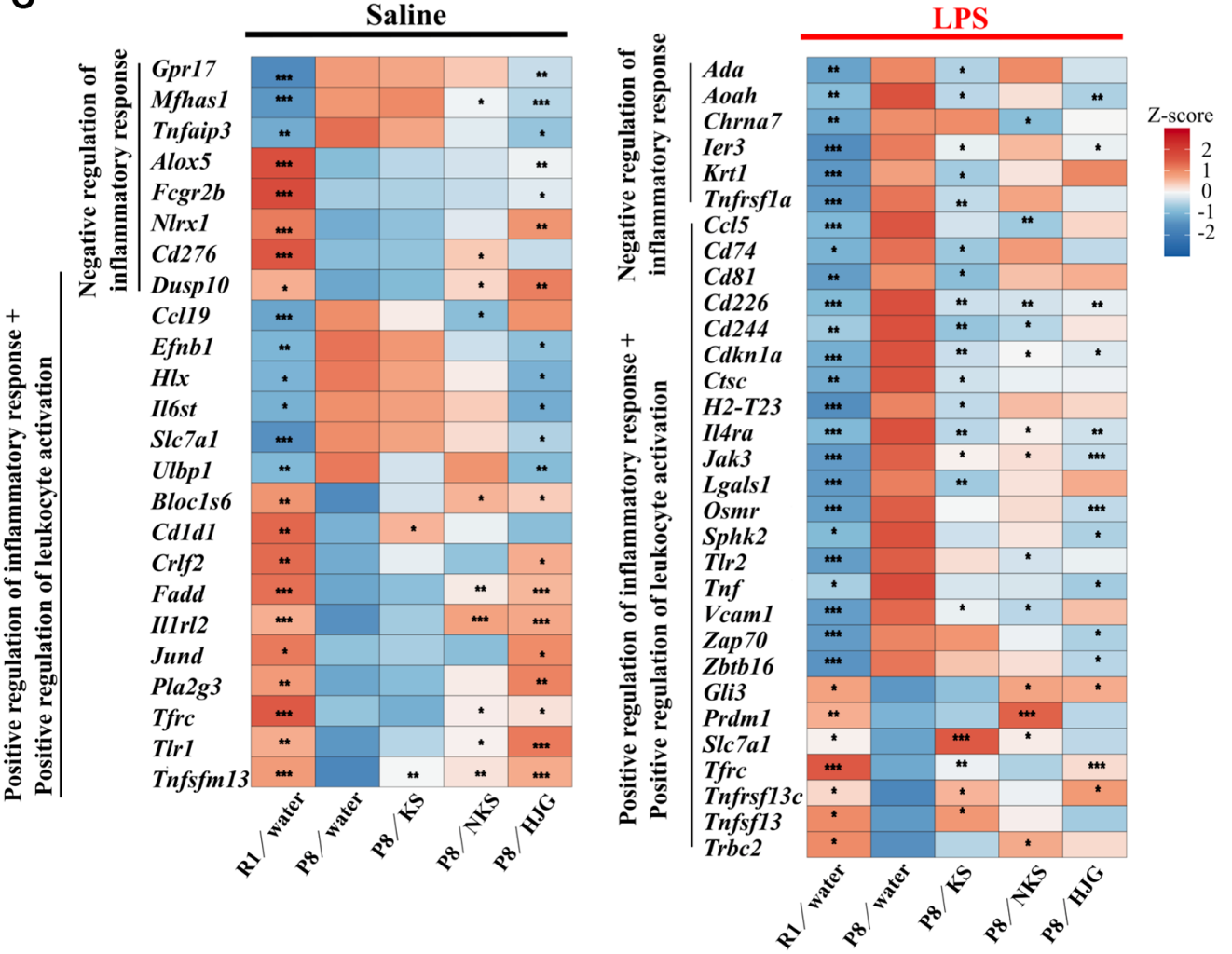

Figure 4. Oral administration of Kampo formulas results in characteristic gene expression patterns in the hippocampus of 19-week-old SAMP8 mice. (A) Genes that were significantly down-regulated in the water-administered SAMP8 mice as compared with water-administered SAMR1 mice and that were significantly up-regulated by treatment with Kampo formulas, i.e., the green line, are referred to as V-shaped recovered genes. Genes that were significantly up-regulated in the water-administered SAMP8 mice as compared with water-administered SAMR1 mice and that were significantly down-regulated by treatment with Kampo formulas, i.e., the orange line, are referred to as reverse $V$-shaped recovered genes. (B) Venn diagrams showing differences and similarities in gene expression among Kampo formula-treated groups. Numbers in circles indicate the number of genes with statistically significant changes in expression $(P<$ 0.05). The full list of genes depicted using lower-case letters is shown in Supplementary Table 1. (C) Heatmap of differential gene expression related to the inflammatory response in the hippocampus of SAMR1 and SAMP8 mice. ${ }^{*} P<0.05,{ }^{* *} P<0.01$, and ${ }^{* * *} P<0.001(n=$ 5) vs. water-administered SAMP8 mice, using the likelihood ratio test. Abbreviations: KS: kososan; NKS: nobiletin-rich kososan; HJG: hachimijiogan; LPS: lipopolysaccharide. 
show that there were many genes that were significantly recovered by each Kampo formula. Among the Kampo formulas, the greatest number of genes, with or without annotation, was altered by HJG treatment (Supplementary Figure 5).

To explore the biological significance of these recovered DEGs, we used the DAVID functional annotation tool. GO analysis of the genes recovered by each Kampo formula was performed, and the top 25 statistically significant (likelihood ratio test, $P<0.05$ ) terms are shown in Supplementary Figure 6. The group of genes that were recovered by KS treatment showed almost no significantly enriched GO and KEGG terms. In contrast, the group of genes that were recovered after treatment with $\mathrm{HJG}$ had the most enriched GO and KEGG terms. Among the Vshaped recovered genes, under physiological conditions, the most enriched GO term was chemotaxis, for both NKS and HJG treatment (Supplementary Figure 6A). In terms of the reverse $\mathrm{V}$-shaped recovered genes, the mostenriched GO terms were related to the regulation of transcription (Supplementary Figure 6B). In contrast, under LPS-stimulated conditions, the V-shaped recovered genes showed that the most enriched GO term was bone mineralization in KS treatment and cell cycle in HJG treatment, while few enriched GO terms were shown in NKS treatment (Supplementary Figure 6C). In terms of the reverse V-shaped recovered genes, the most-enriched GO terms for all KS, NKS and HJG treatment were related to the immune system (Supplementary Figure 6D). Several terms related to response to virus and interferon response, as well as other immune-related terms, were significantly enriched by HJG treatment.

A KEGG pathway analysis revealed that signaling pathways related to various cellular functions (e.g., cell survival, proliferation, apoptosis, cancer) were more enriched in both V-shaped (Supplementary Figure 6E and 6G) and reverse $\mathrm{V}$-shaped recovered genes (Supplementary Figure 6F and 6H) associated with HJG treatment. Additionally, in the reverse $\mathrm{V}$-shaped recovered genes, virus-related terms were also observed under LPS stimulation in the groups that received KS or HJG treatment (Supplementary Figure 6H). These results indicate that LPS-induced immune, inflammatory, and viral responses in the hippocampus were downregulated by treatment with Kampo formulas, and with $\mathrm{HJG}$ in particular.

Next, we focused on the recovered transcripts classified into the GO category "positive and negative regulation of inflammatory response" and "positive regulation of leukocyte activation." Under physiological conditions, significant differences in numerous genes related to the inflammatory response were found between wateradministered SAMR1 and water-administered SAMP8 mice, and these were significantly regulated by treatment with Kampo formulas, particularly HJG (Figure 4C, left). Similarly, under LPS-stimulated conditions, significant differences in numerous genes related to the inflammatory response were found between water-administered SAMR1 and water-administered SAMP8 mice, but there were a number of genes that were significantly recovered by treatment with KS, NKS, and HJG (Figure 4C, right). These results imply that each Kampo formula induces a characteristic gene expression pattern in the hippocampus of SAMP8 mice.

\section{Hippocampal tau levels are suppressed by KS and NKS treatment in SAMP8 mice}

Depression with mild cognitive impairment is associated with progression to Alzheimer's disease [56]. Consequently, we examined the influence of Kampo formulas on tau levels in the hippocampus of 19-weekold SAMP8 mice. Hippocampal tau levels were significantly higher in the water-administered SAMP8 mice than in the water-administered SAMR1 mice (Figure 5A and 5B, Bonferroni's post-hoc test, $P<0.01$ ). These levels were significantly reduced by treatment with KS and NKS (Bonferroni's post-hoc test; KS, $P<0.05$; NKS, $P<0.01)$. However, no change was found between groups in terms of tau synthesis per se, as indicated by the gene expression levels of Mapt, the gene encoding tau (Figure 5C). These results suggest that tau accumulation, but not its synthesis, is hindered by treatment with KS and NKS. This finding is consistent with observations made in coronal brain sections (Supplementary Figure 7), suggesting that KS and NKS exert a suppressive effect on tau accumulation in the brain.

Next, to identify the potential sites of action of Kampo formulas for abrogating tau accumulation, we assessed the expression of genes involved in tau phosphorylation (Figure 5D). Cdkrapl, a gene encoding an inhibitor of cyclin-dependent protein kinase 5 (Cdk5) [57] that phosphorylates tau, showed V-shaped recovered expression with KS treatment. The gene encoding dualspecificity tyrosine phosphorylated and regulated kinase 2 (Dyrk2) [58] showed reverse V-shaped recovered expression with HJG treatment. NKS and HJG treatment both caused reverse $\mathrm{V}$-shaped recovered of the genes encoding Krüppel-like factor-10 (Klf10) and Ngfi-Abinding protein-2 (Nab2), which mediate neurotoxicity and tau phosphorylation, respectively [59]. Furthermore, expression of the gene encoding TRA2B (Tra2b), a splicing factor that contributes to alternative splicing of tau exon 10 in the central nervous system, was also reversed with NKS and HJG treatment. The alternative usage of exon 10 results in tau isoforms containing either three or four microtubule-binding repeats (3R-tau and 4R-tau, respectively) [60]. Thus, changes in Tra2b 
expression in SAMP8 mice may affect the 3R-tau/4Rtau expression ratio.

Autophagy is a degradation pathway involved in the clearance of various toxic aggregated proteins associated with neurodegenerative diseases, such as tau in tauopathies [61]. Therefore, we searched for genes related to autophagy regulation among the recovered genes. As shown in Figure 5E, several autophagy- related genes were detected among both V-shaped and reverse V-shaped recovered genes associated with NKS and HJG treatment. These results suggest that reduction of tau protein caused by KS and NKS treatment is more likely due to inhibition of tau phosphorylation than to tau degradation by autophagy. However, it remains uncertain why HJG failed to reduce tau levels, regardless of marked changes in genes related to tau dynamics.

A

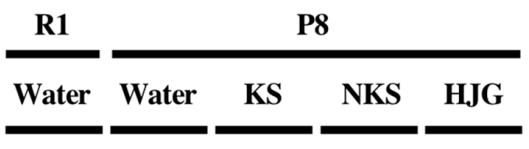

Tau

GAPDH

B

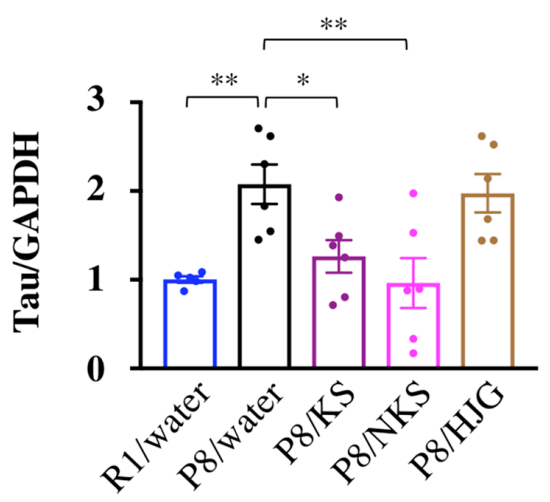

D

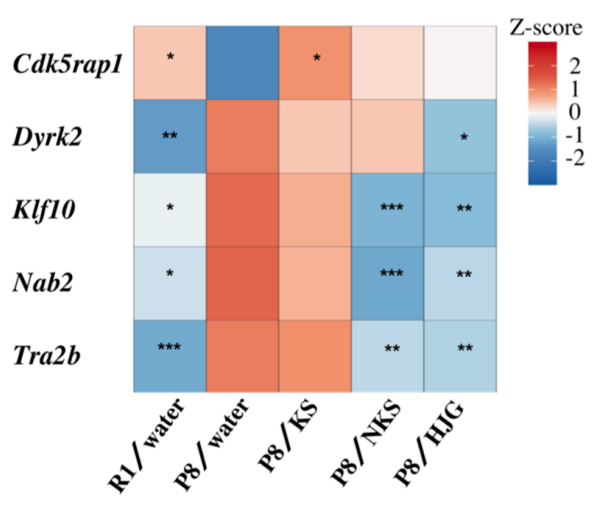

C

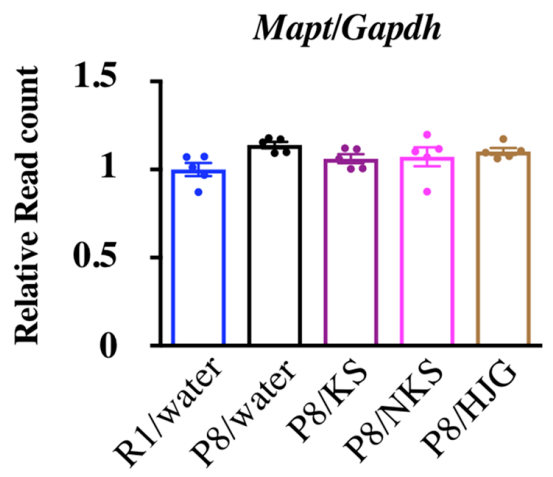

E

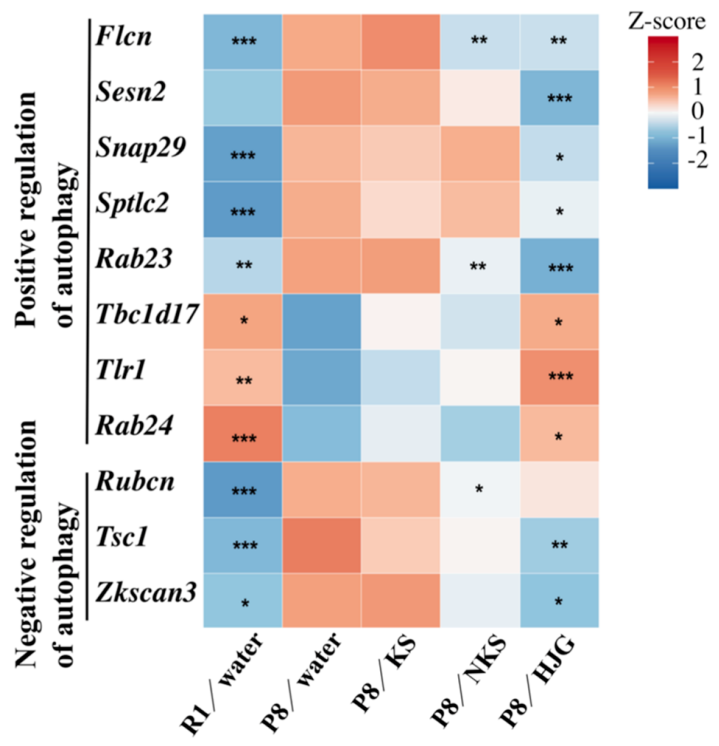

Figure 5. Oral administration of KS and NKS blocks the increase in the level of tau observed in the hippocampus of SAMP8 mice. (A) Representative western blot images of tau and GAPDH expression. (B) Expression of tau was normalized based on GAPDH expression. (C) Expression of Mapt was normalized based on Gapdh expression. Heatmap of differential gene expression related to phosphorylation and splicing of tau (D) and autophagy (E) in the hippocampus of mice. Data are shown as the mean $\pm \operatorname{SEM}(n=5$ or 6$) .{ }^{*} P<$ $0.05,{ }^{* *} P<0.01$, and ${ }^{* * *} P<0.001$ using Bonferroni's post-hoc test. Abbreviations: KS: kososan; NKS: nobiletin-rich kososan; HJG: hachimijiogan. 


\section{Hippocampal levels of pro- and anti-inflammatory cytokines and chemokines are modulated by NKS treatment}

To verify the effects of Kampo formulas on hippocampal neuroinflammation in western blotting analysis, we measured hippocampal protein levels of pro- and anti-inflammatory cytokines and chemokines by ELISA. Under physiological conditions, IL-1 $\beta$ and IL-6 levels were significantly reduced in the NKSadministered SAMP8 mice as compared with the wateradministered SAMP8 mice (Figure 6; Bonferroni's post-hoc test; IL-1 $\beta, P<0.01$; IL-6, $P<0.05)$. The NKS-administered SAMP8 mice also showed a tendency for reduced MCP-1 levels as compared with the water-administered SAMP8 mice (Bonferroni's post-hoc test, $P<0.1)$. Under LPS-stimulated conditions, IL-1 $\beta$ and MCP-1 levels were significantly reduced in the NKS-administered SAMP8 mice as compared with the water-administered SAMP8 mice (Bonferroni's post-hoc test, IL-1 $\beta, P<0.05$; MCP-1, $P$ $<0.05)$. The NKS-administered SAMP8 mice also showed a trend toward elevated IL-4 levels as compared with the water-administered SAMP8 mice (Bonferroni's post-hoc test, $P<0.1)$. These results partially reflected data obtained from western blotting analysis, particularly in the NKS-treated group, which suggested that NKS plays a role in preventing hippocampal neuroinflammation and neuroinflammatory priming, thereby leading to antidepressant-like effects.

\section{Oral administration of HJG suppresses systemic inflammation in SAMP8 mice}

To examine whether Kampo formulas affect systemic inflammation, serum levels of inflammatory factors were measured by ELISA. Under LPS-stimulated conditions, serum IL-6 levels were significantly increased in the water-administered SAMP8 mice as compared with the water-administered SAMR1 mice (Figure 7; Dunnett's post-hoc test, $P<0.05$ ), and this increase was significantly blocked by HJG treatment (Dunnett's post-hoc test, $P<0.05$ ). Likewise, the HJGadministered SAMP8 mice showed a significant decrease in serum MCP-1 levels as compared with the water-administered SAMP8 mice (Dunnett's post-hoc test, $P<0.05)$. However, no significant difference in either IL-6 or MCP-1 levels was found between these groups under saline-injected conditions. These results suggest that HJG suppresses systemic inflammation stimulated by LPS.

\section{DISCUSSION}

In the present study, we demonstrated that aginginduced depressive-like behaviors in SAMP8 mice were significantly prevented by treatment with KS, NKS, and HJG. We also found that KS and NKS exerted anti-neuroinflammatory effects and that HJG exerted systemic anti-inflammatory effects, either of which could result in the behavioral recovery of depression. Furthermore, the suppressive effect of NKS on neuroinflammation is likely to be superior to that of KS, as indicated by hippocampal levels of cytokines and chemokines associated with each treatment. RNA-seq analysis revealed unique and characteristic gene expression patterns in the hippocampus of SAMP8 mice treated with each Kampo formula. These findings highlight that Kampo formulas could be used as preventive therapy for depression induced by aging and could provide effective therapeutic strategies for aging -related emotional disturbances.

Our findings suggest that the antidepressant-like effect of KS in SAMP8 mice was due to mitigation of neuroinflammation that resulted from augmentation of the anti-inflammatory response without affecting the inflammatory response. This effect is consistent with our previous finding that the antidepressant-like effects of KS are associated with attenuation of neuroinflammation in socially defeated mice [39]. Thus, enhancement of anti-inflammatory responses by KS may be a mechanism underlying behavioral recovery. Although there was no difference in the antidepressant-like properties between KS and NKS, the inhibitory effects of NKS on neuroinflammation appeared to be superior to those of KS, particularly under LPS-stimulated conditions, as indicated by Western blotting analyses and by cytokine and chemokine levels in the hippocampus. In addition, local elevation of MCP-1, which is produced by microglia [62] and astrocytes [63] under inflammatory conditions in brain microenvironmental niches, is involved in trafficking and recruitment of peripheral monocytes into the brain and thus leads to exacerbation of neuroinflammation [64, 65]. Thus, it could be assumed that NKS exerts antineuroinflammatory effects not only via direct actions against glial cells but also via indirect actions such as the reduced recruitment of peripheral immune cells, including monocytes. It is also presumed that these effects of NKS could be supported by the antineuroinflammatory activity of nobiletin $[40,41,66]$.

In our study, a comparative analysis of the components of KS and NKS revealed that NKS contained substantially higher levels not only of nobiletin but also of sinensetin and tangeretin, which are also polymethoxyflavones, relative to KS (Supplementary Table 2). Nobiletin reduces MCP-1 (also known as chemokine ligand 2, CCL2) production under IL- 
$1 \beta$-stimulated conditions in vitro [67]. Sinensetin and tangeretin also exert anti-neuroinflammatory effects in vitro [68-70]. These findings appear to corroborate the synergistic anti-neuroinflammatory effect of polymethoxyflavones in NKS, but confirmation of this mechanism requires further investigation.
Unlike KS and NKS, HJG protected against systemic inflammation, rather than neuroinflammation, in response to LPS. Blocking IL-6 signaling in the periphery, but not in the brain, exerts rapid and long-lasting antidepressantlike effects in a mouse model of depression [71]. In a clinical trial, subcutaneous injection of tocilizumab, an
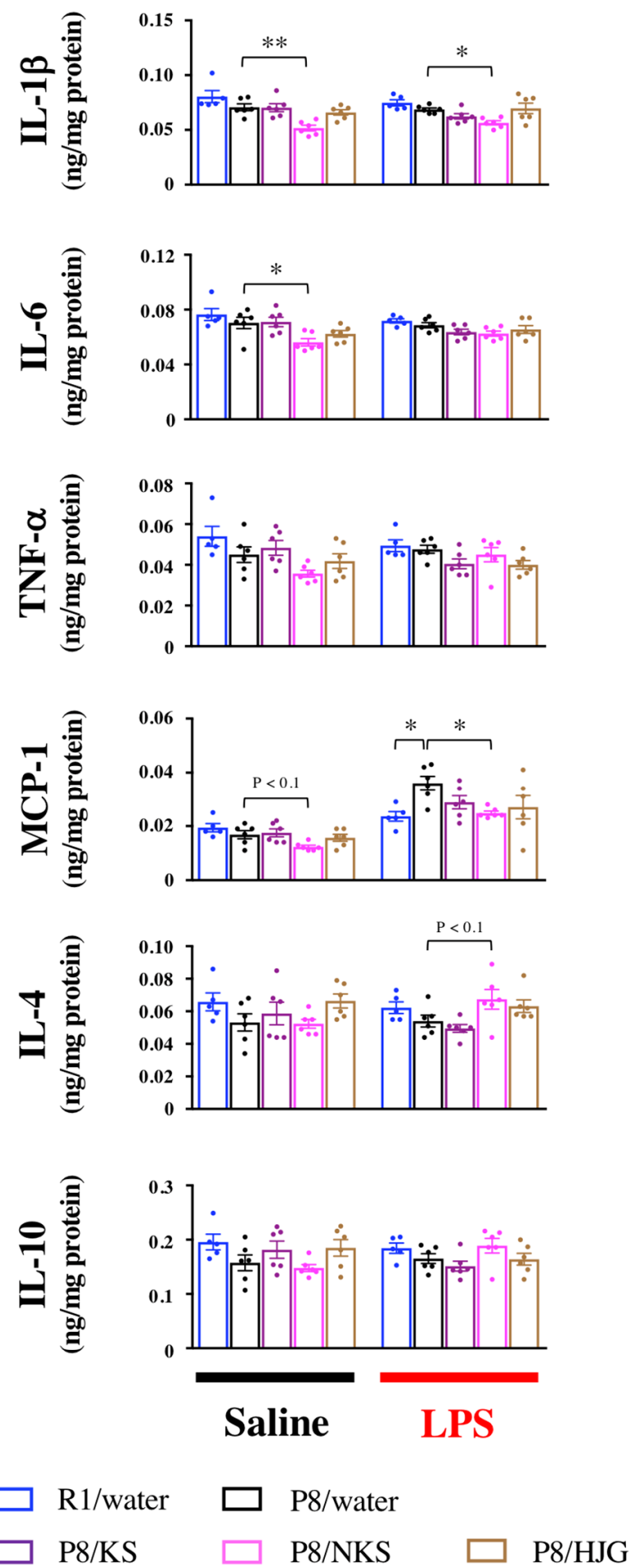

Figure 6. Oral administration of NKS modulates pro- and anti-inflammatory cytokine and chemokine levels in the hippocampus of mice after saline or LPS injection. Data are shown as the mean \pm SEM $(n=5$ or 6$)$. ${ }^{*} P<0.05$ and ${ }^{* *} P<0.01$ using Bonferroni's post-hoc test. Abbreviations: KS: kososan; NKS: nobiletin-rich kososan; HJG: hachimijiogan; LPS: lipopolysaccharide. 
IL-6 antagonist, significantly decreased depression and anxiety levels in patients with rheumatoid arthritis [72]. Given these findings, our results suggest that systemic anti-inflammatory activity underlies the antidepressantlike effect of HJG.

We note that serum levels of CCL2/MCP-1 were reported to be lower in patients with major depressive disorder than in healthy controls [73]. This finding is seemingly inconsistent with our results, which showed that HJG prevented the LPS-induced increase in serum MCP-1 levels, although no difference was found between SAMR1 and SAMP8 mice. Although further studies are needed to verify the biological significance of a reduction in serum MCP-1 levels by treatment with HJG, our results suggest that HJG had a suppressive effect on whole-body inflammation under LPSstimulated conditions, and it may partially underlie the antidepressant-like effect of HJG. Given that NKS was more effective than HJG in its antidepressant-like properties, the effects of inhibiting neuroinflammation may have a greater influence on preventing aginginduced depression than the effects of preventing systemic inflammation, although this requires further verification.

RNA-seq analysis revealed different patterns of gene expression in the hippocampus that were associated with the effects of administration of different Kampo formulas in SAMP8 mice. These gene expression profiles suggest that Kampo formulas possess distinct mechanisms that underlie their effects on behavioral recovery. We expected that functional analysis using GO and KEGG of the recovered genes (Supplementary Figure 6) would reveal specific functions for each Kampo formula; however, because of the small number of recovered genes associated with KS treatment, it was

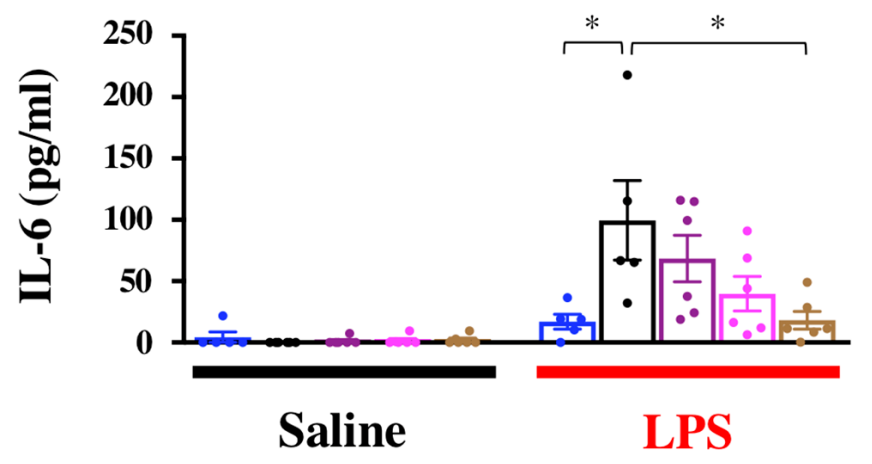

$\mathrm{R} 1 /$ water
D8/water not possible to define clear differences in enriched terms among Kampo formulas. In contrast, HJG treatment resulted in the highest number of recovered genes and thus yielded the most enriched terms. However, different terms were obtained in the V-shaped and reverse $\mathrm{V}$-shaped recovery, and in the physiological state and LPS-treated state, indicating the broad complexity of the influence of this Kampo formula. Under the LPS condition, genes that were recovered by these three Kampo formulas were significantly enriched in the following GO terms: "immune system process," "innate immune response," and "inflammatory response." The expression levels of these genes varied both positively and negatively in SAMP8 mice (Figure 4C). However, these fluctuations in SAMP8 mice were restored by Kampo formulas to approach those in SAMR1 mice, suggesting that Kampo formulas regulate the imbalance of the inflammation system that is associated with aging.

Interestingly, about one-third of the genes, particularly those with V-shaped recovery, were unannotated genes (Supplementary Figure 5). Some of the unannotated genes were significantly positively or negatively correlated with immobility in the TST (Supplementary Table 3). It is likely that among these unannotated genes are some with therapeutic potential associated with the pathogenesis of aging-associated depression, which highlights the need for further gene verification. In addition, significant alterations in epigenetic-related genes were found in the hippocampus of SAMP8 mice relative to SAMR1 mice (Supplementary Figure 8). Moreover, the expression of some genes (Hdacl and Dnmt3a) was recovered by HJG treatment, which may lead to greater dynamics of hippocampal genes related to the inflammatory responses in HJG-administered SAMP8 mice. It is possible that some unannotated

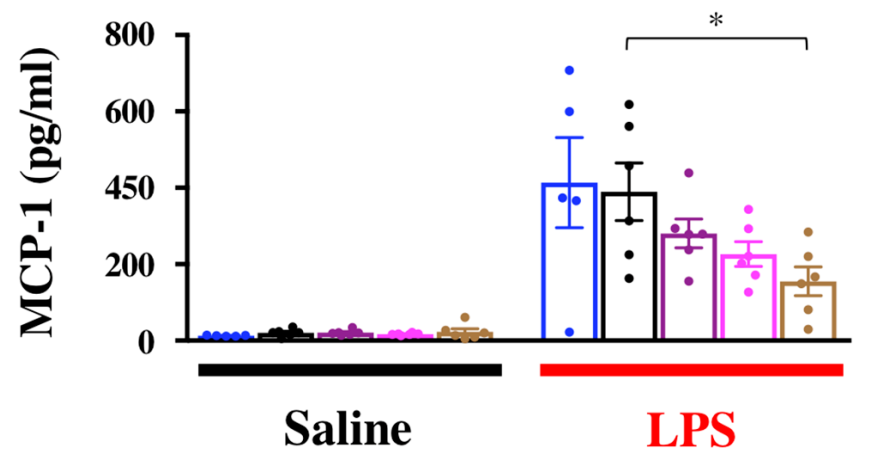

$\mathrm{P} 8 / \mathrm{KS}$
P8/NKS

P8/HJG

Figure 7. Serum levels of a pro-inflammatory cytokine (IL-6) and chemokine (MCP-1) are suppressed by oral administration of HJG after LPS injection. Data are shown as the mean \pm SEM ( $n=5$ or 6$)$. ${ }^{*} P<0.05$ using Dunnett's post-hoc test. Abbreviations: KS: kososan; NKS: nobiletin-rich kososan; HJG: hachimijiogan; LPS: lipopolysaccharide. 
genes underwent significant recovery in response to treatment with Kampo formulas may be involved in these observations. Further studies are required to verify this possibility.

More recently, it was demonstrated by positron emission tomography that accumulation of tau, rather than that of amyloid- $\beta$, in the brain may underlie the manifestation of major depressive disorder (MDD) in older patients [74]. Importantly, no cognitive impairment was found in patients with MDD with increased tau deposition. Given that depression is a risk factor for neurodegenerative diseases, including cognitive dysfunction and dementia [75, 76], it is plausible that depression with tau accumulation precedes the emergence of cognitive impairment. In our study, 19-week-old SAMP8 mice showed tau accumulation in the brain. This level was reduced, in conjunction with behavioral recovery, by KS and NKS treatment. Our findings suggest that reduction in tau accumulation by treatment with KS and NKS may be a possible mechanism underlying the antidepressant-like activities of these Kampo formulas.

In SAMP8 mice at more than 6 months old, nobiletin reduces the phosphorylation of tau, which protects against cognitive dysfunction [77]. This is consistent with our finding that NKS downregulates hippocampal genes involved in tau phosphorylation. However, it is presumed that the effect of nobiletin alone cannot explain the effects of KS and NKS on tau levels. Future studies are required to determine how KS and NKS decrease tau accumulation and how their effects contribute to the antidepressant-like properties.

We also found that some genes relevant to autophagy, phosphorylation, and splicing of tau in the hippocampus were altered by KS, NKS, and HJG treatment in our RNA-seq analysis. Further studies are necessary to clarify why the genes altered by HJG did not result in reduced tau accumulation.

In the present study, LPS injection was used to evaluate neuroinflammatory priming with aging [21, 78-80]. Several findings have been reported that expression of TLR4, which is a well-known innate immune receptor for LPS, is elevated with aging [81, 82]. Given that no differences between SAMP8 and SAMR1 mice aged 19 weeks were observed in the TLR4 expression, it would be presumed that SAMP8 mice aged 19 weeks possess the neuroinflammatory priming rather than intense neuroinflammation along with upregulation of TLR4 expression. The LPS challenge also appears to mimic a state of stress exposure, as assumed by a notable finding showing that social stress induces neuroinflammation via binding of endogenous ligands to TLR4 [83].
Therefore, it is likely that alleviating effects of Kampo formulas on the neuroinflammatory priming in SAMP8 mice may lead to their antidepressant-like activities in the TST, which itself is a stressful behavioral test. Additionally, infections can increase the risk of developing mood disorders, including depression, in humans [84]. Given this finding, the antineuroinflammatory effects of Kampo formulas may lead to a decreased risk of post-infection depression. This possibility could be supported by further studies investigating whether Kampo formulas indeed result in behavioral recovery after LPS injection.

There are several limitations to this study. First, we focused on comparing the effects of the three Kampo formulas on aging-related emotional disturbances and neuroinflammation in SAMP8 mice and found that they exerted preventive effects against depression-like behaviors as well as neuroinflammation associated with aging. Some antidepressants have been reported to attenuate neuroinflammation in vivo [24, 85] and in vitro [86-89], as well as in human studies [25]. Further studies comparing the effects of these Kampo formulas with those of existing antidepressants are necessary for proper evaluation of the efficacy of Kampo medication as a treatment for late-life depression. Second, we did not perform behavioral testing to assess cognitive impairment in 19-week-old SAMP8 mice, because we focused on the effects of Kampo formulas on emotional disturbances before the onset of cognitive dysfunction in these mice. In many cases, SAMP8 mice over 30 weeks of age have been used for evaluating cognitive dysfunction [90-94]. The preventive effects of KS and NKS on tau accumulation in our study may indicate prophylaxis for aging-related cognitive dysfunction, but this requires further verification in aged SAMP8 mice using cognitive tasks. Third, although nobiletin can act as a circadian amplitude enhancer [95, 96], NKS treatment failed to regulate circadian rhythm disruption in SAMP8 mice. A possible explanation for this is that the nobiletin content in NKS was insufficient to reverse circadian rhythm disruption. A second explanation may be that circadian rhythm disruption in SAMP8 mice is genetically and tightly regulated. Additional studies of the effect of NKS on circadian rhythm disruption induced by normal aging are needed to resolve this issue. Fourth, we focused on hippocampal dynamics related to neuroinflammation in this study, but increasing evidence has demonstrated that microglia have brain region-dependent diversity and regionspecific sensitivities to aging [97-100]. Therefore, further comparative studies examining the microglial function specific to emotion-related brain regions (e.g., the hippocampus, amygdala, and prefrontal cortex) in SAMP8 mice could yield a better understanding of the mechanisms underlying aging-related emotional 
disturbances as well as the active sites of each Kampo formula. Fifth, considerable differences in the findings of western blotting and RNA-seq analyses were found in this study. Although this may be related to a temporal gap in the expression of proteins or genes, the effects of unannotated genes detected in this study cannot be disregarded. Another possibility is that there may be some differences in the period for emergence of behavioral recovery between Kampo formulas, which could involve a lag in the expression of genes and proteins. Further studies are required to investigate this possibility. Last, we used SAMP8 mice to examine the effects of Kampo formulas, because these mice show emotional disturbances at relatively young age [101]. However, future studies would be needed to verify the beneficial effects of Kampo formulas in the wild-type aged mice.

\section{CONCLUSIONS}

Aging predisposes individuals to neuroinflammation and emotional disorders, negatively impacting health and reducing the quality of life in older individuals. Given that depression serves as a risk factor for cognitive impairment in late life, preventive therapy would be a more effective strategy for prophylaxis of cognitive decline and other neurodegenerative diseases. Our findings implicate that each Kampo formula has a characteristic efficacy in terms of some different modes of action for neuroinflammation and systemic inflammation and may provide promising options for preventing late-life depression in humans by using Kampo medication. We anticipate further elucidation of the therapeutic role of these Kampo formulas for emotional disorders associated with aging. Since little has been also reported on the comparative study of some Kampo formulas regarding aging-related emotional disturbances, the unique effects of Kampo formulas would be scientifically and clinically informative for therapeutic strategies of aging-related emotional disorders. In addition, further evidence of NKS, which is not clinically used yet in Japan, would be helpful for NKS being available for aging-related depression in humans in the future.

\section{MATERIALS AND METHODS}

\section{Animals}

Male SAMP8 and SAMR1 mice were obtained at 5 weeks of age from Japan SLC (Hamamatsu, Japan) and were allowed to acclimate for 1 week after arrival. Mice were individually housed for avoiding fights and their concomitant injuries at a constant temperature $(23 \pm$ $\left.2{ }^{\circ} \mathrm{C}\right)$ and humidity $(55 \pm 10 \%)$ and with a 12-h light/dark cycle (lights on at 08:00) with access to food
(LabDiet5058, Lab Supply, Fort Worth, TX, USA) and water ad libitum.

All animal experiments were approved by the Institutional Animal Care and Use Committee of Kitasato University and were performed in accordance with the Guidelines for the Care and Use of Laboratory Animals of Kitasato University and the National Research Council Guide for the Care and Use of Laboratory Animals in Japan. Every effort was made to minimize the number of animals used and their suffering.

\section{Preparation of Kampo extract}

The component herbs of KS, NKS, and HJG are shown in Table 1. Each Kampo formula was decocted with 600 $\mathrm{ml}$ of distilled water until the volume was reduced by half. The water extract was immediately filtered with a commercial tea strainer and centrifuged at $1000 \times g$ for $10 \mathrm{~min}$ at $4^{\circ} \mathrm{C}$, and the supernatant was lyophilized. The total yield of $\mathrm{KS}, \mathrm{NKS}$, and $\mathrm{HJG}$ extracts was approximately $28 \%, 25 \%$, and $19.6 \%$, respectively, relative to the dry weight of the herbal mixture. The preparation of KS and NKS extracts and their component analysis were performed by Kotaro Pharmaceutical Co., Ltd (Osaka, Japan).

\section{Drug treatment and measurement of body weight}

Each extract of the Kampo formula was dissolved in distilled water. The extract $(1.0 \mathrm{~g} / \mathrm{kg}$ body weight) or distilled water was administered once daily by oral gavage to mice from 7 weeks of age for 13 weeks (Figure 8). The dose of KS extract in this study was selected based on our previous studies, which showed that KS extract has an antidepressant-like effect in mouse models of stress-induced depression [35-37, 39, 102]. Other Kampo extracts (NKS and HJG) were set to the same dose as KS for comparison. The dose of each Kampo formula used in mice was equivalent to approximately 15 -fold of that in humans. Body weight was measured in mice once a week before the drug treatment.

\section{Monitoring of locomotor activity}

Locomotor activity of mice in their home cages was simultaneously recorded for $24 \mathrm{~h}$ using a Nanotag device (Kissei Comtec Co., Ltd., Nagano, Japan), an implantable three-axis accelerometer (weight, $2.6 \mathrm{~g}$ ). The nanotag was intraperitoneally implanted in half of mice per group aged 6 weeks under inhalation anesthesia with isoflurane (Figure 8). After recovery for 1 week, the locomotor activity of mice at 7, 9, 11, 13, 15 , and 19 weeks of age was recorded in 4-min bins 
Table 1. Component herbs and daily quantities for kososan (KS), nobiletin-rich kososan (NKS), and hachimijiogan (HJG).

\begin{tabular}{lccc}
\hline \multicolumn{1}{c}{ Herb } & KS & NKS & HJG \\
\hline a. Cyperus rhizome & $4 \mathrm{~g}$ & $4 \mathrm{~g}$ & \\
b. Perilla herb & $2 \mathrm{~g}$ & $2 \mathrm{~g}$ & \\
c. Citrus unshiu peel & $3 \mathrm{~g}$ & & \\
d. Citrus reticulata peel & & $3 \mathrm{~g}$ & \\
e. Glycyrrhiza & $2 \mathrm{~g}$ & $2 \mathrm{~g}$ & \\
f. Ginger & $0.5 \mathrm{~g}$ & $0.5 \mathrm{~g}$ & $6 \mathrm{~g}$ \\
g. Rehmannia root & & & $3 \mathrm{~g}$ \\
h. Alisma rhizome & & $3 \mathrm{~g}$ \\
i. Poria sclerotium & & $3 \mathrm{~g}$ \\
j. Dioscorea rhizome & & $3 \mathrm{~g}$ \\
k. Cornus fruit & & & $3 \mathrm{~g}$ \\
1. Moutan bark & & & $1 \mathrm{~g}$ \\
m. Cinnamon bark & & $1 \mathrm{~g}$ \\
n. Aconite root & $11.5 \mathrm{~g}$ & $23 \mathrm{~g}$ \\
\hline Total herbal weight per day & & $11.5 \mathrm{~g}$ \\
\hline
\end{tabular}

Herbs (a-c, f) were purchased from Tsumura and Co. (Tokyo, Japan). Herb (d) was purchased from Kotaro Pharmaceutical Co., Ltd. (Osaka, Japan). Herbs (e, g, h, n) were purchased from Uchida Wakan-yaku Co., Ltd. (Tokyo, Japan). Herbs (i, j, k, I, m) were purchased from Tochimoto Tenkaido Co., Ltd. (Tokyo, Japan).

over $24 \mathrm{~h}$, and the data were analyzed using the Nanotag viewer program (Kissei Comtec Co., Ltd.).

\section{Open field test}

For the open field test (OFT), individual mice were introduced into an opaque gray open field box $(40 \times 40$ $\times 40 \mathrm{~cm}$ ) and were allowed to explore freely for $5 \mathrm{~min}$ under high light conditions (120-130 lux). Time spent in three zones (center, intermediate, and peripheral zones) and the total distance traveled were recorded using a video tracking system (EthoVision 3.0; Noldus, Wageningen, Netherlands). Anxiety-like behaviors were evaluated based on the time spent in the three zones. The OFT was performed between 12:00 and 17:00 after acclimation to a habituation room for $1 \mathrm{~h}$.

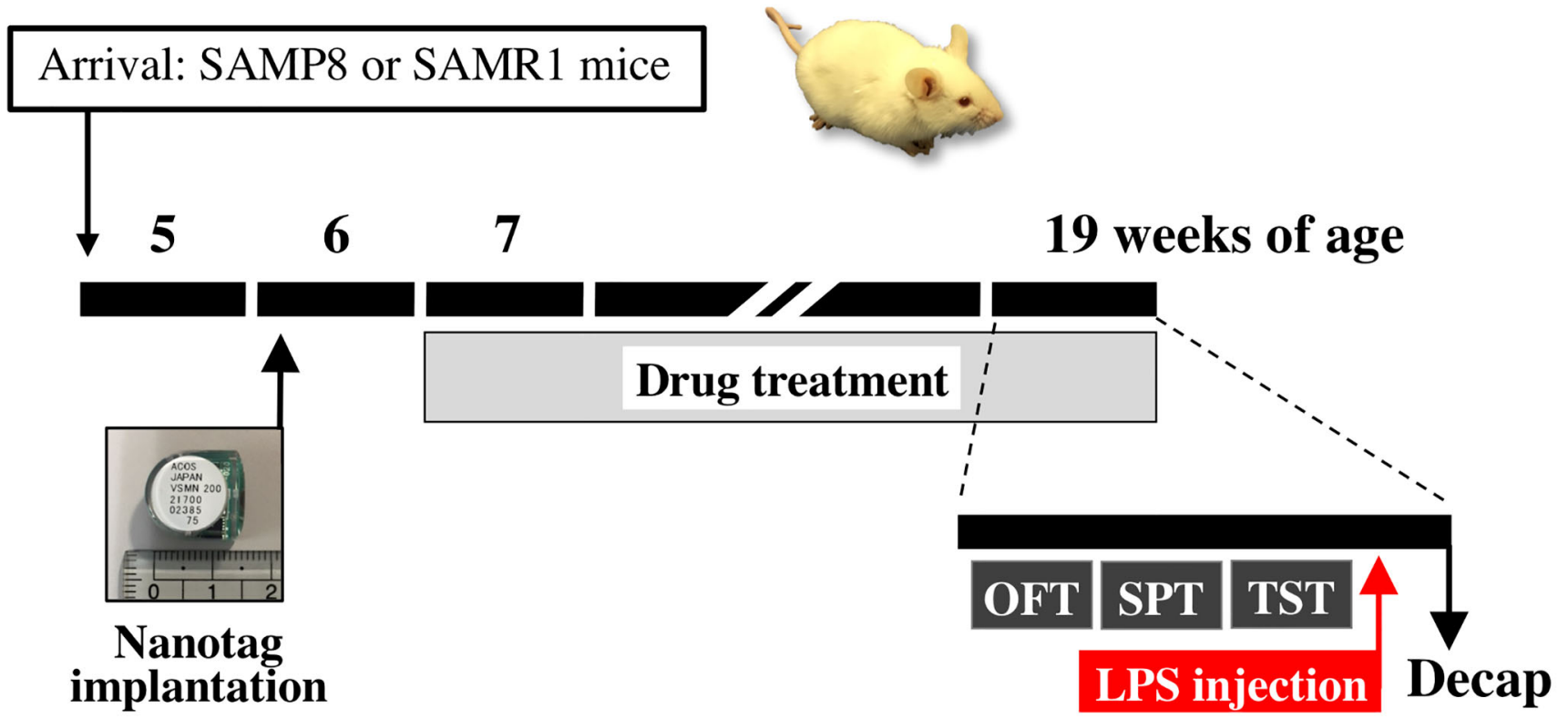

Figure 8. Schematic representation of the experimental schedule. Abbreviations: OFT: open field test; SPT: sucrose preference test; TST: tail suspension test; Decap: decapitation; LPS: lipopolysaccharide. 


\section{Sucrose preference test}

The sucrose preference test (SPT) was performed to evaluate anhedonia, a depression-like behavior, as described [21, 103] with some modifications. Briefly, mice were trained to adapt to the presentation of two bottles of water in their home cages for 2 days. After adaptation, each mouse had free access to two bottles, one containing a $2 \%$ sucrose solution and the other containing water, with a counterbalance in its home cage from 17:00 to 9:00 over 2 days. The sucrose preference ratio was calculated as follows: ( $2 \%$ sucrose solution intake $[\mathrm{ml}] /$ total fluid intake $[\mathrm{ml}]) \times 100$, and the average of the ratio per mouse over the 2 days of the experiment was used for analysis.

\section{Tail suspension test}

The tail suspension test (TST) was performed to evaluate apathy, which is a depression-like behavior, as described [21, 104] with some modifications. Briefly, for $6 \mathrm{~min}$ the mice were suspended by their tails $50 \mathrm{~cm}$ above the floor using experimental clips (Yamashitagiken, Tokushima, Japan) connected to a hook that was connected to a steel bar. A mouse was considered immobile only when it ceased struggling and hung motionless. All behaviors were videotaped, and the duration of immobility during the last $4 \mathrm{~min}$ of the TST was determined. The TST was conducted between 12:00 and 14:00 after acclimation to a habituation room for $1 \mathrm{~h}$.

\section{Lipopolysaccharide injection}

After the TST, mice were injected i.p. with lipopolysaccharide (LPS, serotype O55:B5, $0.33 \mathrm{mg} / \mathrm{kg}$ body weight; Sigma, St. Louis, MO, USA) or saline. Intraperitoneal LPS injection was performed at a volume of $10 \mathrm{ml} / \mathrm{kg}$ body weight.

\section{Brain and serum collection}

Twenty-four hours after LPS or saline injection, the mice were decapitated, and their brains and trunk blood samples were collected. The brains were frozen in liquid nitrogen. Two hours after blood sampling, trunk blood was centrifuged twice at $3,000 \times g$ for $1 \mathrm{~min}$ at $4^{\circ} \mathrm{C}$ to collect serum. Brain and serum samples were stored at $-80^{\circ} \mathrm{C}$ until further processing.

\section{Western blotting}

Frozen brains were sliced into $\sim 1$-mm-thick coronal sections (bregma -1.5 to $-2.5 \mathrm{~mm}$ ), and hippocampi were dissected on dry ice. Proteins were extracted from the hippocampi by sonication of the tissue samples in lysis buffer (CelLytic MT, Sigma) containing 1\% (v/v) protease inhibitor cocktail (Sigma) on ice, followed by centrifugation at $18,000 \times g$ for $10 \mathrm{~min}$ at $4^{\circ} \mathrm{C}$ and collection of the resulting supernatants. Total protein content was determined using a BCA protein assay kit (Pierce, Rockford, IL, USA). Proteins $(10 \mu \mathrm{g})$ were separated on 4-15\% SDS-PAGE gels (Bio-Rad, Tokyo, Japan) and were transferred to polyvinylidene difluoride membranes (Bio-Rad) using a Trans-Blot Turbo $^{\mathrm{TM}}$ Transfer System (Bio-Rad). After being blocked with Blocking One solution (Nacalai Tesque, Kyoto, Japan) for $30 \mathrm{~min}$ at room temperature (RT), the membranes were incubated with the following primary antibodies at RT for $1 \mathrm{~h}$ or $4^{\circ} \mathrm{C}$ overnight, as indicated: rabbit antinod-like receptor family, pyrin domain-containing (NLRP)3 (1:1000, overnight; Cell Signaling Technology, Tokyo, Japan), rabbit anti-arginase (Arg) 1 (1:3000, overnight; GeneTex, Irvine, CA, USA), rat anti-tau (1:2000, overnight; Fujifilm Wako Pure Chemical Corporation, Osaka, Japan), or horseradish peroxidase (HRP)-conjugated mouse anti-GAPDH (1:10000, $1 \mathrm{~h}$; Proteintech, Rosemont, IL, USA). The membranes were then rinsed in Tris-buffered saline containing $0.05 \%(\mathrm{v} / \mathrm{v})$ Tween 20 and were incubated with appropriate HRP-linked secondary antibodies (except in the case of anti-GAPDH) for $1 \mathrm{~h}$ at RT. The blots were visualized with Clarity Western ECL Substrate or Clarity Max Western ECL Substrate (BioRad) using a ChemiDoc ${ }^{\mathrm{TM}}$ Imaging System (ChemiDoc Touch, Bio-Rad). Band intensities were analyzed using ImageLab software (Ver. 6.0, Bio-Rad).

\section{RNA extraction and RNA-sequencing}

For RNA extraction, frozen brains were sliced into $\sim 1$ $\mathrm{mm}$-thick coronal sections (bregma -2.5 to $-3.5 \mathrm{~mm}$ ), and the hippocampi were dissected on dry ice. Samples were immersed in RNAlater solution (Thermo Fisher Scientific, Carlsbad, CA, USA) and cut into small pieces $(\sim 30 \mathrm{mg}$ each) using scissors. RNA extraction was performed using a Pure Link RNA Mini kit (Invitrogen, Carlsbad, CA, USA). To this end, $300 \mu \mathrm{L}$ of lysis buffer and $450 \mu \mathrm{L}$ of TRIzol-LS (Thermo Fisher Scientific) were added to the tissue. The tissue was then homogenized on ice using a BioMasher disposable homogenizer (Nippi, Tokyo, Japan). After the sample was incubated for $10 \mathrm{~min}$ at RT, it was centrifuged at $12,000 \times g$ for $15 \mathrm{~min}$. The supernatant was treated with DNase and the resultant RNA was purified using spin columns. The quality of the total RNA was assessed using a Qubit 4.0 (Thermo Fisher Scientific) and a 4200TapeStation system (Agilent Technologies, Santa Clara, CA, USA). Each fresh-frozen total RNA sample had a RNA Integrity Number $($ RIN) $>7$, indicating that they were of sufficient quality to prepare sequencing libraries. 
Total RNA was used for RNA-sequencing (RNA-seq) analysis. Library preparation and Illumina HiSeq sequencing were conducted by GENEWIZ (South Plainfield, NJ, USA), which generated $\sim 20$ million (150-basepair, paired-end) reads per library.

\section{Quality check and filtering of RNA-seq data and mapping analysis}

For quality filtering of the RNA-seq data, cutadapt v.1.16 [105] was used to remove Illumina adapter sequences, followed by removal of the poly-A sequence using fastx_clipper software in the fastx toolkit software package v.0.0.14 (FASTX-Toolkit, http://hannonlab. cshl.edu/fastx toolkit/index.html). To remove lowquality bases or sequences, we trimmed the sequences using fastq quality_trimmer software (parameters: -t 20 $\left.\begin{array}{llll}-1 & 30 & -Q & 33\end{array}\right)$ and fastq quality_filter software (parameters: -q 20 -p $80-\mathrm{Q}$ 33), both of which were included in the fastx toolkit. During the above processing, any read in which one of the pairs was missing was removed using Trimmomatic v.0.38 [106]. Then, reads containing mouse rRNA, tRNA, or phiX sequences, which are the control sequences from Illumina, were removed using Bowtie 2 v. 2.3.4.1 [107]. We then performed a second round of removal of any unpaired reads using bam2fastq (https://gslweb. discoveryls.com/information/software/bam2fastq).

After completion of these filtering steps, 20 million reads of each of the forward and reverse sequences per sample were mapped to the mouse genome sequence to build GRCm38, using HISAT2 v2.1.0 [108]. The mouse genome sequence was downloaded from Illumina iGenomes (https://support.illumina.com/sequencing/ sequencing software/igenome.html). Multiple-mapped reads were removed using samtools (parameters: samtools view -q 4) [109]. Uniquely mapped reads were counted by gene annotation (Ensembl release 81) using featureCounts v.1.6.2 [110]. The counted values were normalized using the Trimmed mean of $M$ values (TMM) method [111] using the edgeR library [112] in $\mathrm{R} \quad$ v.3.5.0 (https://cran.r-project.org/bin/windows/ base/old/3.5.0/) and were used for expression analysis. The resultant RNA-seq data were subjected to multidimensional scaling analysis. The original RNAseq datasets were deposited in the DDBJ Sequence Read Archive under accession numbers DRR310804DRR310853 which are linked to the BioProject accession number PRJDB015423.

\section{Analysis of differentially expressed genes}

$P$-values for the differentially expressed genes (DEGs) were obtained using the likelihood ratio test in the edgeR (https://cran.r-project.org/bin/windows/base/old/3.5.0/) package. First, we extracted genes whose expression levels were significantly down- or up-regulated $(P<$ $0.05)$ in the water-administered SAMP8 mice (SAMP8/water) as compared with the wateradministered SAMR1mice (SAMR1/water). Among the genes whose expression levels were significantly downregulated (SAMR1/water > SAMP8/water), those showing a pattern of significant up-regulation in Kampo- administered SAMP8 mice (SAMP8/Kampo; $\mathrm{KS}$, NKS, or HJG) as compared with SAMP8/water were referred to as "V-shaped recovered" genes (SAMR1/water > SAMP8/water, and SAMP8/water < SAMP8/Kampo) (Figure 4A, green line). Conversely, among the genes whose expression levels were significantly up-regulated (SAMR1/water < SAMP8/water), those that showed a pattern of significant down-regulation in SAMP8/Kampo as compared with SAMP8/water were referred to as "reverse V-shaped recovered" genes (SAMR1/water < SAMP8/water, and SAMP8/water > SAMP8/Kampo) (Figure 4A, orange line).

\section{Gene ontology analysis and pathway analysis}

The DEGs were mapped to the relevant biological annotation from Gene Ontology (GO; http://www. geneontology.org/) and the Kyoto Encyclopedia of Genes and Genomes (KEGG) database (https://www. genome.jp/kegg/) using the DAVID functional annotation online database v.6.8 (https://david. ncifcrf.gov/). Fisher's exact test was applied to identify significant GO terms and pathways, and the threshold of significance was defined by the $P$-value and false discovery rate.

\section{Enzyme-linked immunosorbent assay}

Cytokine and chemokine levels in the samples were detected using a commercially available enzyme-linked immunosorbent assay (ELISA) kit for interleukin (IL)$1 \beta$ and IL-6 (BD OptEIA ${ }^{\mathrm{TM}}$ ELISA set, BD Biosciences, San Diego, CA, USA) and for tumor necrosis factor (TNF)- $\alpha$, IL-4, IL-10, and monocyte chemoattractant protein (MCP)-1 (Mouse DuoSet ELISA, R\&D Systems, Minneapolis, MN, USA).

\section{Statistical analysis}

All data are presented as the mean \pm SEM and were analyzed using Prism 7 (GraphPad Software, San Diego, CA, USA). We used the paired $t$-test for comparisons between two groups. Data were also analyzed by one-way ANOVA, followed by Bonferroni's or Dunnett's post-hoc test for comparisons among multiple groups. Correlation analysis was also performed using Pearson's 
correlation coefficient. For gene expression analysis based on RNA-seq, $P$-values were obtained using the likelihood ratio test. In all cases, $P<0.05$ was considered statistically significant.

\section{Abbreviations}

Arg 1: Arginase1; Cdk5: Cyclin-dependent protein kinase 5; DEGs: differentially expressed genes; Dyrk2: Dual-specificity tyrosine phosphorylated and regulated kinase 2; GO: Gene Ontology; HJG: Hachimijiogan; HRP: Horseradish peroxidase; IL: Interleukin; KEGG: Kyoto Encyclopedia of Genes and Genomes; Klf10: Krüppel-like factor-10; KS: Kososan; LPS: Lipopolysaccharide; MCP-1: Monocyte chemoattractant protein-1; MDD: Major depressive disorder; Nab2: Ngfi-A-binding protein-2; NKS: Nobiletin-rich kososan; NLRP3: Nod-like receptor family, pyrin domain-containing 3; OFT: Open field test; RIN: RNA integrity number; RNAseq: RNA-sequencing; RT: Room temperature; SAMP8: Senescence-accelerated mouse prone 8; SAMR1: Senescence-accelerated mouse resistant 1; SPT: Sucrose preference test; TLR4: Toll-like receptor 4; TNF- $\alpha$ : Tumor necrosis factor- $\alpha$; TST: Tail suspension test.

\section{AUTHOR CONTRIBUTIONS}

NI and AM designed and performed the experiments, analyzed the data, and drafted the manuscript. KO helped with RNA-seq experiments. MY prepared KS and NKS extracts and performed their component analysis. $\mathrm{KH}$ and $\mathrm{CS}$ assisted with behavioral experiments. $\mathrm{TN}, \mathrm{YK}, \mathrm{HO}$, and $\mathrm{NO}$ discussed the results and edited the manuscript. All authors read and approved the final manuscript.

\section{ACKNOWLEDGMENTS}

We thank Dr. Haruki Yamada for his advice on this research. We would like to thank Ms. Harumi Koguchi for her technical assistance. We are grateful to Ms. Kano Koike, Mr. Tomonori Tsuda, Mr. Takahiko Sakagami, and Mr. Hiroyoshi Kikuta for their financial support through crowdfunding.

\section{CONFLICTS OF INTEREST}

NI, TN, YK, and $\mathrm{HO}$ declare financial conflicts of interest from Tsumura \& Co. Norihiro Okada's research group (AM, KO, and NO) has an ongoing collaboration with Tsumura \& Co., and this collaboration has included financial support. Tsumura \& Co. had no role in the design of the research, analysis or interpretation of the results, or in writing the manuscript.

\section{FUNDING}

This work was supported by JSPS KAKENHI (grant number $17 \mathrm{~K} 09320$ and $21 \mathrm{~K} 07376$ to NI), JST COI (Grant number JPMJCE1301 to NI and HO), and crowdfunding (NI).

\section{REFERENCES}

1. Campos Costa I, Nogueira Carvalho H, Fernandes L. Aging, circadian rhythms and depressive disorders: a review. Am J Neurodegener Dis. 2013; 2:228-46. PMID:24319642

2. Alexopoulos GS. Mechanisms and treatment of latelife depression. Transl Psychiatry. 2019; 9:188. https://doi.org/10.1038/s41398-019-0514-6 PMID:31383842

3. Gambino CM, Sasso BL, Bivona G, Agnello L, Ciaccio M. Aging and Neuroinflammatory Disorders: New Biomarkers and Therapeutic Targets. Curr Pharm Des. 2019; 25:4168-74.

https://doi.org/10.2174/1381612825666191112093034 PMID:31721696

4. Landi F, Calvani R, Tosato M, Martone AM, Ortolani E, Savera G, Sisto A, Marzetti E. Anorexia of Aging: Risk Factors, Consequences, and Potential Treatments. Nutrients. 2016; 8:69.

https://doi.org/10.3390/nu8020069

PMID:26828516

5. Halim M, Halim A. The effects of inflammation, aging and oxidative stress on the pathogenesis of diabetes mellitus (type 2 diabetes). Diabetes Metab Syndr. 2019; 13:1165-72.

https://doi.org/10.1016/j.dsx.2019.01.040

PMID: $\underline{31336460}$

6. Pietri P, Stefanadis C. Cardiovascular Aging and Longevity: JACC State-of-the-Art Review. J Am Coll Cardiol. 2021; 77:189-204. https://doi.org/10.1016/i.jacc.2020.11.023 PMID:33446313

7. Fathi Kazerooni A, Pozo JM, McCloskey EV, Saligheh Rad H, Frangi AF. Diffusion MRI for Assessment of Bone Quality; A Review of Findings in Healthy Aging and Osteoporosis. J Magn Reson Imaging. 2020; 51:975-92.

https://doi.org/10.1002/jmri.26973 PMID:31709670

8. Lafosse E, Wolffsohn JS, Talens-Estarelles C, GarcíaLázaro S. Presbyopia and the aging eye: Existing refractive approaches and their potential impact on dry eye signs and symptoms. Cont Lens Anterior Eye. 2020; 43:103-14. https://doi.org/10.1016/i.clae.2019.08.005 
PMID: $\underline{31445772}$

9. Gates GA, Mills JH. Presbycusis. Lancet. 2005; 366:1111-20.

https://doi.org/10.1016/S0140-6736(05)67423-5

PMID:16182900

10. Larsson L, Degens H, Li M, Salviati L, Lee Yl, Thompson W, Kirkland JL, Sandri M. Sarcopenia: Aging-Related Loss of Muscle Mass and Function. Physiol Rev. 2019; 99:427-511.

https://doi.org/10.1152/physrev.00061.2017 PMID: 30427277

11. Furman D, Campisi J, Verdin E, Carrera-Bastos P, Targ S, Franceschi C, Ferrucci L, Gilroy DW, Fasano A, Miller GW, Miller AH, Mantovani A, Weyand CM, et al. Chronic inflammation in the etiology of disease across the life span. Nat Med. 2019; 25:1822-32.

https://doi.org/10.1038/s41591-019-0675-0

PMID:31806905

12. Di Benedetto S, Müller L, Wenger E, Düzel S, Pawelec G. Contribution of neuroinflammation and immunity to brain aging and the mitigating effects of physical and cognitive interventions. Neurosci Biobehav Rev. 2017; 75:114-28.

https://doi.org/10.1016/j.neubiorev.2017.01.044 PMID:28161508

13. Pizza V, Agresta A, D'Acunto CW, Festa M, Capasso A. Neuroinflamm-aging and neurodegenerative diseases: an overview. CNS Neurol Disord Drug Targets. 2011; 10:621-34.

https://doi.org/10.2174/187152711796235014

PMID:21631404

14. Ransohoff RM. How neuroinflammation contributes to neurodegeneration. Science. 2016; 353:777-83.

https://doi.org/10.1126/science.aag2590

PMID:27540165

15. Holbrook JA, Jarosz-Griffiths $H H$, Caseley E, LaraReyna S, Poulter JA, Williams-Gray CH, Peckham D, McDermott MF. Neurodegenerative Disease and the NLRP3 Inflammasome. Front Pharmacol. 2021; 12:643254.

https://doi.org/10.3389/fphar.2021.643254

PMID:33776778

16. Yirmiya R, Rimmerman N, Reshef R. Depression as a microglial disease. Trends Neurosci. 2015; 38:637-58. https://doi.org/10.1016/j.tins.2015.08.001 PMID:26442697

17. Mondelli V, Vernon AC, Turkheimer F, Dazzan P, Pariante CM. Brain microglia in psychiatric disorders. Lancet Psychiatry. 2017; 4:563-72. https://doi.org/10.1016/S2215-0366(17)30101-3 PMID:28454915
18. Pfau ML, Ménard C, Russo SJ. Inflammatory Mediators in Mood Disorders: Therapeutic Opportunities. Annu Rev Pharmacol Toxicol. 2018; 58:411-28.

https://doi.org/10.1146/annurev-pharmtox-010617052823

PMID:28992428

19. Woelfer M, Kasties V, Kahlfuss S, Walter M. The Role of Depressive Subtypes within the Neuroinflammation Hypothesis of Major Depressive Disorder. Neuroscience. 2019; 403:93-110. https://doi.org/10.1016/j.neuroscience.2018.03.034 PMID:29604382

20. Meyer JH, Cervenka S, Kim MJ, Kreisl WC, Henter ID, Innis RB. Neuroinflammation in psychiatric disorders: PET imaging and promising new targets. Lancet Psychiatry. 2020; 7:1064-74.

https://doi.org/10.1016/S2215-0366(20)30255-8 PMID:33098761

21. Ito $\mathrm{N}$, Takemoto $\mathrm{H}$, Hasegawa A, Sugiyama C, Honma K, Nagai T, Kobayashi $Y$, Odaguchi $H$. Neuroinflammaging underlies emotional disturbances and circadian rhythm disruption in young male senescence-accelerated mouse prone 8 mice. Exp Gerontol. 2020; 142:111109.

https://doi.org/10.1016/j.exger.2020.111109

PMID:33069781

22. Chung YC, Kim SR, Park JY, Chung ES, Park KW, Won SY, Bok E, Jin M, Park ES, Yoon SH, Ko HW, Kim YS, Jin BK. Fluoxetine prevents MPTP-induced loss of dopaminergic neurons by inhibiting microglial activation. Neuropharmacology. 2011; 60:963-74.

https://doi.org/10.1016/j.neuropharm.2011.01.043 PMID:21288472

23. Ramirez K, Shea DT, McKim DB, Reader BF, Sheridan JF. Imipramine attenuates neuroinflammatory signaling and reverses stress-induced social avoidance. Brain Behav Immun. 2015; 46:212-20.

https://doi.org/10.1016/i.bbi.2015.01.016 PMID:25701613

24. Du RH, Tan J, Sun XY, Lu M, Ding JH, Hu G. Fluoxetine Inhibits NLRP3 Inflammasome Activation: Implication in Depression. Int J Neuropsychopharmacol. 2016; 19:pyw037.

https://doi.org/10.1093/ijnp/pyw037 PMID:27207922

25. Almeida IB, Gomes IA, Shanmugam S, de Moura TR, Magalhães LS, de Aquino LAG, de Souza Araújo AA, Oliveira PD, Santos MRV. Inflammatory modulation of fluoxetine use in patients with depression: A systematic review and meta-analysis. Cytokine. 2020; 131:155100. 
https://doi.org/10.1016/j.cyto.2020.155100 PMID: $\underline{32315957}$

26. Andersohn F, Schade R, Suissa S, Garbe E. Long-term use of antidepressants for depressive disorders and the risk of diabetes mellitus. Am J Psychiatry. 2009; 166:591-8.

https://doi.org/10.1176/appi.ajp.2008.08071065 PMID:19339356

27. Pan SJ, Tan YL, Yao SW, Xin Y, Yang X, Liu J, Xiong J. Fluoxetine induces lipid metabolism abnormalities by acting on the liver in patients and mice with depression. Acta Pharmacol Sin. 2018; 39:1463-72. https://doi.org/10.1038/aps.2017.207 PMID:30150788

28. Zhou SF. Drugs behave as substrates, inhibitors and inducers of human cytochrome P450 3A4. Curr Drug Metab. 2008; 9:310-22.

https://doi.org/10.2174/138920008784220664 PMID: 18473749

29. Knadler MP, Lobo E, Chappell J, Bergstrom R. Duloxetine: clinical pharmacokinetics and drug interactions. Clin Pharmacokinet. 2011; 50:281-94. https://doi.org/10.2165/11539240-000000000-00000 PMID:21366359

30. Tatonetti NP, Denny JC, Murphy SN, Fernald GH, Krishnan G, Castro V, Yue P, Tsao PS, Kohane I, Roden DM, Altman RB. Detecting drug interactions from adverse-event reports: interaction between paroxetine and pravastatin increases blood glucose levels. Clin Pharmacol Ther. 2011; 90:133-42.

https://doi.org/10.1038/clpt.2011.83

PMID:21613990

31. Shin J, Hills NK, Finley PR. Combining Antidepressants with $\beta$-Blockers: Evidence of a Clinically Significant CYP2D6 Drug Interaction. Pharmacotherapy. 2020; 40:507-16.

https://doi.org/10.1002/phar.2406

PMID:32342526

32. Oberlin LE, Respino M, Victoria L, Abreu L, Hoptman MJ, Alexopoulos GS, Gunning FM. Late-life depression accentuates cognitive weaknesses in older adults with small vessel disease. Neuropsychopharmacology. 2022; 47:580-7. https://doi.org/10.1038/s41386-021-00973-z PMID:33564103

33. Gabryelewicz T, Styczynska M, Luczywek E, Barczak A, Pfeffer A, Androsiuk W, Chodakowska-Zebrowska M, Wasiak B, Peplonska B, Barcikowska M. The rate of conversion of mild cognitive impairment to dementia: predictive role of depression. Int J Geriatr Psychiatry. 2007; 22:563-7.

https://doi.org/10.1002/gps.1716

PMID:17136705
34. Ito $\mathrm{N}$, Nagai $\mathrm{T}$, Yabe $\mathrm{T}$, Nunome $\mathrm{S}$, Hanawa $\mathrm{T}$, Yamada $\mathrm{H}$. Antidepressant-like activity of a Kampo (Japanese herbal) medicine, Koso-san (Xiang-Su-San), and its mode of action via the hypothalamic-pituitaryadrenal axis. Phytomedicine. 2006; 13:658-67. https://doi.org/10.1016/j.phymed.2006.01.002 PMID: 16516452

35. Nagai T, Narikawa T, Ito N, Takeda T, Hanawa T, Yamada H. Antidepressant-like effect of a Kampo (Japanese herbal) medicine, kososan, against the interferon- $\alpha$-induced depressive-like model mice. J Trad Med. 2008; 25:74-80. https://doi.org/10.11339/jtm.25.74

36. Ito $\mathrm{N}$, Yabe $\mathrm{T}$, Nagai $\mathrm{T}$, Oikawa $\mathrm{T}$, Yamada H, Hanawa T. A possible mechanism underlying an antidepressive-like effect of Kososan, a Kampo medicine, via the hypothalamic orexinergic system in the stress-induced depression-like model mice. Biol Pharm Bull. 2009; 32:1716-22.

https://doi.org/10.1248/bpb.32.1716

PMID:19801833

37. Ito $N$, Hori A, Yabe $T$, Nagai T, Oikawa T, Yamada $H$, Hanawa $T$. Involvement of neuropeptide $Y$ signaling in the antidepressant-like effect and hippocampal cell proliferation induced by kososan, a Kampo medicine, in the stress-induced depression-like model mice. Biol Pharm Bull. 2012; 35:1775-83.

https://doi.org/10.1248/bpb.b12-00466 PMID:23037167

38. Nagai T, Hashimoto R, Okuda SM, Kodera Y, Oh-Ishi M, Maeda T, Ito N, Hanawa T, Kiyohara H, Yamada H. Antidepressive-like effect of a Kampo (traditional Japanese) medicine, kososan (Xiang Su San) in a stress-induced depression-like mouse model: Proteomic analysis of hypothalamus. Trad \& Kampo Med. 2015; 2:50-9. https://doi.org/10.1002/tkm2.1018

39. Ito $N$, Hirose $E$, Ishida $T$, Hori A, Nagai $T$, Kobayashi $Y$, Kiyohara H, Oikawa T, Hanawa T, Odaguchi H. Kososan, a Kampo medicine, prevents a social avoidance behavior and attenuates neuroinflammation in socially defeated mice. J Neuroinflammation. 2017; 14:98. https://doi.org/10.1186/s12974-017-0876-8 PMID:28468634

40. Cui Y, Wu J, Jung SC, Park DB, Maeng YH, Hong JY, Kim SJ, Lee SR, Kim SJ, Kim SJ, Eun SY. Antineuroinflammatory activity of nobiletin on suppression of microglial activation. Biol Pharm Bull. 2010; 33:1814-21. https://doi.org/10.1248/bpb.33.1814 PMID:21048305 
41. Qi G, Mi Y, Fan R, Li R, Liu Z, Liu X. Nobiletin Protects against Systemic Inflammation-Stimulated Memory Impairment via MAPK and NF-kB Signaling Pathways. J Agric Food Chem. 2019; 67:5122-34.

https://doi.org/10.1021/acs.jafc.9b00133 PMID:30995031

42. Cho HW, Jung SY, Lee GH, Cho JH, Choi IY. Neuroprotective effect of Citrus unshiu immature peel and nobiletin inhibiting hydrogen peroxideinduced oxidative stress in HT22 murine hippocampal neuronal cells. Pharmacogn Mag. 2015 (Suppl 2); 11:S284-9.

https://doi.org/10.4103/0973-1296.166047 PMID:26664016

43. Wang H, Guo Y, Qiao Y, Zhang J, Jiang P. Nobiletin Ameliorates NLRP3 Inflammasome-Mediated Inflammation Through Promoting Autophagy via the AMPK Pathway. Mol Neurobiol. 2020; 57:5056-68.

https://doi.org/10.1007/s12035-020-02071-5 PMID:32833187

44. Yi LT, Xu HL, Feng J, Zhan X, Zhou LP, Cui CC. Involvement of monoaminergic systems in the antidepressant-like effect of nobiletin. Physiol Behav. 2011; 102:1-6.

https://doi.org/10.1016/i.physbeh.2010.10.008 PMID:20951716

45. Li J, Zhou Y, Liu BB, Liu Q, Geng D, Weng LJ, Yi LT. Nobiletin Ameliorates the Deficits in Hippocampal BDNF, TrkB, and Synapsin I Induced by Chronic Unpredictable Mild Stress. Evid Based Complement Alternat Med. 2013; 2013:359682.

https://doi.org/10.1155/2013/359682 PMID:23573124

46. Nakajima A, Yamakuni T, Matsuzaki K, Nakata N, Onozuka H, Yokosuka A, Sashida Y, Mimaki Y, Ohizumi Y. Nobiletin, a citrus flavonoid, reverses learning impairment associated with $\mathrm{N}$-methyl-D-aspartate receptor antagonism by activation of extracellular signal-regulated kinase signaling. J Pharmacol Exp Ther. 2007; 321:784-90.

https://doi.org/10.1124/ipet.106.117010

PMID: 17289833

47. Nakajima A, Aoyama Y, Shin EJ, Nam Y, Kim HC, Nagai T, Yokosuka A, Mimaki Y, Yokoi T, Ohizumi Y, Yamada K. Nobiletin, a citrus flavonoid, improves cognitive impairment and reduces soluble $A \beta$ levels in a triple transgenic mouse model of Alzheimer's disease (3XTg-AD). Behav Brain Res. 2015; 289:69-77.

https://doi.org/10.1016/j.bbr.2015.04.028

PMID:25913833

48. Kawahata I, Yoshida M, Sun W, Nakajima A, Lai Y, Osaka N, Matsuzaki K, Yokosuka A, Mimaki Y, Naganuma A, Tomioka Y, Yamakuni T. Potent activity of nobiletin-rich Citrus reticulata peel extract to facilitate CAMP/PKA/ERK/CREB signaling associated with learning and memory in cultured hippocampal neurons: identification of the substances responsible for the pharmacological action. J Neural Transm (Vienna). 2013; 120:1397-409.

https://doi.org/10.1007/s00702-013-1025-x

PMID:23588349

49. Seki T, Kamiya T, Furukawa K, Azumi M, Ishizuka S, Takayama S, Nagase S, Arai H, Yamakuni T, Yaegashi N. Nobiletin-rich Citrus reticulata peels, a kampo medicine for Alzheimer's disease: a case series. Geriatr Gerontol Int. 2013; 13:236-8. https://doi.org/10.1111/j.1447-0594.2012.00892.x PMID:23286569

50. Iwasaki K, Kobayashi S, Chimura Y, Taguchi M, Inoue K, Cho S, Akiba T, Arai H, Cyong JC, Sasaki H. A randomized, double-blind, placebo-controlled clinical trial of the Chinese herbal medicine "ba wei di huang wan" in the treatment of dementia. J Am Geriatr Soc. 2004; 52:1518-21.

https://doi.org/10.1111/j.1532-5415.2004.52415.x PMID:15341554

51. Kainuma M, Funakoshi K, Ouma S, Yamashita KI, Ohara T, Yoshiiwa A, Murata M, Tsuboi Y. The efficacy and safety of hachimijiogan for mild Alzheimer disease in an exploratory, open standard treatment controlled, randomized allocation, multicenter trial: A study protocol. Medicine (Baltimore). 2020; 99:e22370.

https://doi.org/10.1097/MD.0000000000022370 PMID: $\underline{32957414}$

52. Liu YL, Lee WC. Traditional Chinese medicine and herbal supplements for treating overactive bladder. Urological Science. 2018; 29:216-22. https://doi.org/10.4103/UROS.UROS 818

53. Krishnan V, Nestler EJ. The molecular neurobiology of depression. Nature. 2008; 455:894-902.

https://doi.org/10.1038/nature07455

PMID: 18923511

54. Son $Y$, Yang $M$, Wang $H$, Moon C. Hippocampal dysfunctions caused by cranial irradiation: a review of the experimental evidence. Brain Behav Immun. 2015; 45:287-96.

https://doi.org/10.1016/j.bbi.2015.01.007 PMID:25596174

55. Roddy DW, Farrell C, Doolin K, Roman E, Tozzi L, Frodl T, O'Keane V, O'Hanlon E. The Hippocampus in Depression: More Than the Sum of Its Parts? Advanced Hippocampal Substructure Segmentation in Depression. Biol Psychiatry. 2019; 85:487-97. https://doi.org/10.1016/j.biopsych.2018.08.021 PMID: $\underline{30528746}$ 
56. Defrancesco $M$, Marksteiner J, Kemmler $G$, Fleischhacker WW, Blasko I, Deisenhammer EA. Severity of Depression Impacts Imminent Conversion from Mild Cognitive Impairment to Alzheimer's Disease. J Alzheimers Dis. 2017; 59:1439-48.

https://doi.org/10.3233/JAD-161135

PMID:28731429

57. Ching YP, Pang AS, Lam WH, Qi RZ, Wang JH. Identification of a neuronal Cdk5 activator-binding protein as Cdk5 inhibitor. J Biol Chem. 2002; 277:15237-40.

https://doi.org/10.1074/ibc.C200032200

PMID:11882646

58. Woods YL, Cohen P, Becker W, Jakes R, Goedert M, Wang $X$, Proud CG. The kinase DYRK phosphorylates protein-synthesis initiation factor elF2Bepsilon at Ser539 and the microtubule-associated protein tau at Thr212: potential role for DYRK as a glycogen synthase kinase 3-priming kinase. Biochem J. 2001; 355:609-15. https://doi.org/10.1042/bj3550609

PMID:11311121

59. Killick R, Ribe EM, Al-Shawi R, Malik B, Hooper C, Fernandes C, Dobson R, Nolan PM, Lourdusamy A, Furney S, Lin K, Breen G, Wroe R, et al. Clusterin regulates $\beta$-amyloid toxicity via Dickkopf-1-driven induction of the wnt-PCP-JNK pathway. Mol Psychiatry. 2014; 19:88-98.

https://doi.org/10.1038/mp.2012.163

PMID:23164821

60. Glatz DC, Rujescu D, Tang Y, Berendt FJ, Hartmann AM, Faltraco F, Rosenberg C, Hulette C, Jellinger $K$, Hampel H, Riederer P, Möller HJ, Andreadis A, et al. The alternative splicing of tau exon 10 and its regulatory proteins CLK2 and TRA2-BETA1 changes in sporadic Alzheimer's disease. J Neurochem. 2006; 96:635-44.

https://doi.org/10.1111/j.1471-4159.2005.03552.x PMID:16371011

61. Rubinsztein DC, Bento CF, Deretic V. Therapeutic targeting of autophagy in neurodegenerative and infectious diseases. J Exp Med. 2015; 212:979-90.

https://doi.org/10.1084/jem.20150956

PMID:26101267

62. Cazareth J, Guyon A, Heurteaux C, Chabry J, Petit-Paitel A. Molecular and cellular neuroinflammatory status of mouse brain after systemic lipopolysaccharide challenge: importance of CCR2/CCL2 signaling. J Neuroinflammation. 2014; 11:132.

https://doi.org/10.1186/1742-2094-11-132 PMID:25065370

63. Ménard C, Pfau ML, Hodes GE, Russo SJ. Immune and Neuroendocrine Mechanisms of Stress Vulnerability and Resilience. Neuropsychopharmacology. 2017; 42:62-80.

https://doi.org/10.1038/npp.2016.90

PMID:27291462

64. Wohleb ES, McKim DB, Sheridan JF, Godbout JP. Monocyte trafficking to the brain with stress and inflammation: a novel axis of immune-to-brain communication that influences mood and behavior. Front Neurosci. 2015; 8:447.

https://doi.org/10.3389/fnins.2014.00447 PMID:25653581

65. Weber MD, Godbout JP, Sheridan JF. Repeated Social Defeat, Neuroinflammation, and Behavior: Monocytes Carry the Signal. Neuropsychopharmacology. 2017; 42:46-61. https://doi.org/10.1038/npp.2016.102 PMID:27319971

66. Li W, Wang X, Niu X, Zhang H, He Z, Wang Y, Zhi W, Liu F. Protective Effects of Nobiletin Against Endotoxic Shock in Mice Through Inhibiting TNF- $\alpha$, IL6, and HMGB1 and Regulating NF-KB Pathway. Inflammation. 2016; 39:786-97. https://doi.org/10.1007/s10753-016-0307-5 PMID:26846885

67. Hosokawa Y, Hosokawa I, Ozaki K, Matsuo T. Nobiletin Inhibits Inflammatory Reaction in Interleukin-1ß-Stimulated Human Periodontal Ligament Cells. Pharmaceutics. 2021; 13:667. https://doi.org/10.3390/pharmaceutics13050667 PMID:34066937

68. Shin HS, Kang SI, Yoon SA, Ko HC, Kim SJ. Sinensetin attenuates LPS-induced inflammation by regulating the protein level of IкB- $\alpha$. Biosci Biotechnol Biochem. 2012; 76:847-9. https://doi.org/10.1271/bbb.110908 PMID:22484952

69. Laavola M, Nieminen R, Yam MF, Sadikun A, Asmawi MZ, Basir R, Welling J, Vapaatalo H, Korhonen R, Moilanen E. Flavonoids eupatorin and sinensetin present in Orthosiphon stamineus leaves inhibit inflammatory gene expression and STAT1 activation. Planta Med. 2012; 78:779-86.

https://doi.org/10.1055/s-0031-1298458 PMID:22516932

70. Shu Z, Yang B, Zhao H, Xu B, Jiao W, Wang Q, Wang Z, Kuang $\mathrm{H}$. Tangeretin exerts anti-neuroinflammatory effects via NF-KB modulation in lipopolysaccharidestimulated microglial cells. Int Immunopharmacol. 2014; 19:275-82. https://doi.org/10.1016/j.intimp.2014.01.011 PMID:24462494

71. Zhang JC, Yao W, Dong C, Yang C, Ren Q, Ma M, 
Hashimoto K. Blockade of interleukin- 6 receptor in the periphery promotes rapid and sustained antidepressant actions: a possible role of gutmicrobiota-brain axis. Transl Psychiatry. 2017; 7:e1138.

https://doi.org/10.1038/tp.2017.112 PMID:28556833

72. Tiosano S, Yavne Y, Watad A, Langevitz P, Lidar M, Feld J, Tishler M, Aamar S, Elkayam O, Balbir-Gurman A, Molad Y, Ehrlich S, Abu-Shakra M, et al. The impact of tocilizumab on anxiety and depression in patients with rheumatoid arthritis. Eur J Clin Invest. 2020; 50:e13268.

https://doi.org/10.1111/eci.13268

PMID: $\underline{2478417}$

73. Lehto SM, Niskanen L, Herzig KH, Tolmunen T, Huotari A, Viinamäki $H$, Koivumaa-Honkanen $H$, Honkalampi K, Ruotsalainen H, Hintikka J. Serum chemokine levels in major depressive disorder. Psychoneuroendocrinology. 2010; 35:226-32. https://doi.org/10.1016/j.psyneuen.2009.06.007 PMID:19592174

74. Moriguchi S, Takahata K, Shimada H, Kubota M, Kitamura S, Kimura $\mathrm{Y}$, Tagai $\mathrm{K}$, Tarumi R, Tabuchi $\mathrm{H}$, Meyer JH, Mimura M, Kawamura K, Zhang MR, et al. Excess tau PET ligand retention in elderly patients with major depressive disorder. Mol Psychiatry. 2020. [Epub ahead of print]. https://doi.org/10.1038/s41380-020-0766-9 PMID:32606373

75. Byers AL, Yaffe K. Depression and risk of developing dementia. Nat Rev Neurol. 2011; 7:323-31. https://doi.org/10.1038/nrneurol.2011.60 PMID:21537355

76. Diniz BS, Butters MA, Albert SM, Dew MA, Reynolds CF 3rd. Late-life depression and risk of vascular dementia and Alzheimer's disease: systematic review and meta-analysis of community-based cohort studies. Br J Psychiatry. 2013; 202:329-35. https://doi.org/10.1192/bjp.bp.112.118307 PMID:23637108

77. Nakajima A, Aoyama Y, Nguyen TT, Shin EJ, Kim HC, Yamada S, Nakai T, Nagai T, Yokosuka A, Mimaki Y, Ohizumi Y, Yamada K. Nobiletin, a citrus flavonoid, ameliorates cognitive impairment, oxidative burden, and hyperphosphorylation of tau in senescenceaccelerated mouse. Behav Brain Res. 2013; 250:351-60.

https://doi.org/10.1016/i.bbr.2013.05.025 PMID:23714077

78. Frank MG, Barrientos RM, Watkins LR, Maier SF. Aging sensitizes rapidly isolated hippocampal microglia to LPS ex vivo. J Neuroimmunol. 2010;
226:181-4.

https://doi.org/10.1016/i.jneuroim.2010.05.022 PMID:20537730

79. Garner KM, Amin R, Johnson RW, Scarlett EJ, Burton MD. Microglia priming by interleukin- 6 signaling is enhanced in aged mice. J Neuroimmunol. 2018; 324:90-9.

https://doi.org/10.1016/i.jneuroim.2018.09.002 PMID: $\underline{0261355}$

80. Fonken LK, Frank MG, Gaudet AD, Maier SF. Stress and aging act through common mechanisms to elicit neuroinflammatory priming. Brain Behav Immun. 2018; 73:133-48. https://doi.org/10.1016/j.bbi.2018.07.012 PMID: $\underline{30009999}$

81. Letiembre M, Hao W, Liu Y, Walter S, Mihaljevic I, Rivest S, Hartmann T, Fassbender K. Innate immune receptor expression in normal brain aging. Neuroscience. 2007; 146:248-54.

https://doi.org/10.1016/i.neuroscience.2007.01.004 PMID:17293054

82. Calvo-Rodríguez $M$, de la Fuente $C$, García-Durillo $M$, García-Rodríguez C, Villalobos C, Núñez L. Aging and amyloid $\beta$ oligomers enhance TLR4 expression, LPSinduced $\mathrm{Ca}^{2+}$ responses, and neuron cell death in cultured rat hippocampal neurons. J Neuroinflammation. 2017; 14:24.

https://doi.org/10.1186/s12974-017-0802-0 PMID:28143556

83. Nie $X$, Kitaoka $S$, Tanaka $K$, Segi-Nishida E, Imoto $Y$, Ogawa A, Nakano F, Tomohiro A, Nakayama K, Taniguchi M, Mimori-Kiyosue Y, Kakizuka A, Narumiya $S$, Furuyashiki T. The Innate Immune Receptors TLR2/4 Mediate Repeated Social Defeat StressInduced Social Avoidance through Prefrontal Microglial Activation. Neuron. 2018; 99:464-79.e7. https://doi.org/10.1016/j.neuron.2018.06.035 PMID:30033154

84. Benros ME, Waltoft BL, Nordentoft M, Ostergaard SD, Eaton WW, Krogh J, Mortensen PB. Autoimmune diseases and severe infections as risk factors for mood disorders: a nationwide study. JAMA Psychiatry. 2013; 70:812-20.

https://doi.org/10.1001/jamapsychiatry.2013.1111 PMID:23760347

85. Ramirez K, Sheridan JF. Antidepressant imipramine diminishes stress-induced inflammation in the periphery and central nervous system and related anxiety- and depressive- like behaviors. Brain Behav Immun. 2016; 57:293-303.

https://doi.org/10.1016/i.bbi.2016.05.008

PMID:27223094 
86. Tynan RJ, Weidenhofer J, Hinwood M, Cairns MJ, Day TA, Walker FR. A comparative examination of the anti-inflammatory effects of SSRI and SNRI antidepressants on LPS stimulated microglia. Brain Behav Immun. 2012; 26:469-79.

https://doi.org/10.1016/j.bbi.2011.12.011 PMID:22251606

87. Liu RP, Zou M, Wang JY, Zhu JJ, Lai JM, Zhou LL, Chen SF, Zhang $\mathrm{X}$, Zhu JH. Paroxetine ameliorates lipopolysaccharide-induced microglia activation via differential regulation of MAPK signaling. J Neuroinflammation. 2014; 11:47.

https://doi.org/10.1186/1742-2094-11-47

PMID:24618100

88. Fujimori K, Takaki J, Shigemoto-Mogami Y, Sekino Y, Suzuki T, Sato K. Paroxetine prevented the downregulation of astrocytic L-Glu transporters in neuroinflammation. J Pharmacol Sci. 2015; 127:145-9. https://doi.org/10.1016/j.jphs.2014.09.002 PMID:25704030

89. Zhang $X$, Zhu LB, He JH, Zhang $\mathrm{HQ}$, Ji SY, Zhang CN, Hou NN, Huang CP, Zhu JH. Paroxetine suppresses reactive microglia-mediated but not lipopolysaccharide-induced inflammatory responses in primary astrocytes. J Neuroinflammation. 2020; 17:50. https://doi.org/10.1186/s12974-020-1712-0 PMID:32024542

90. Maurice T, Roman FJ, Su TP, Privat A. Beneficial effects of sigma agonists on the age-related learning impairment in the senescence-accelerated mouse (SAM). Brain Res. 1996; 733:219-30.

https://doi.org/10.1016/0006-8993(96)00565-3

PMID: 8891305

91. Zhang X, Li G, Guo L, Nie K, Jia Y, Zhao L, Yu J. Agerelated alteration in cerebral blood flow and energy failure is correlated with cognitive impairment in the senescence-accelerated prone mouse strain 8 (SAMP8). Neurol Sci. 2013; 34:1917-24.

https://doi.org/10.1007/s10072-013-1407-8 PMID:23563860

92. Yanai S, Toyohara J, Ishiwata K, Ito H, Endo S. Longterm cilostazol administration ameliorates memory decline in senescence-accelerated mouse prone 8 (SAMP8) through a dual effect on CAMP and bloodbrain barrier. Neuropharmacology. 2017; 116:247-59. https://doi.org/10.1016/i.neuropharm.2016.12.006 PMID:27979612

93. Puigoriol-Illamola D, Griñán-Ferré C, Vasilopoulou $F$, Leiva R, Vázquez S, Pallàs M. 11 $\beta$-HSD1 Inhibition by RL-118 Promotes Autophagy and Correlates with Reduced Oxidative Stress and Inflammation, Enhancing Cognitive Performance in SAMP8 Mouse Model. Mol Neurobiol. 2018; 55:8904-15. https://doi.org/10.1007/s12035-018-1026-8 PMID:29611102

94. Currais A, Huang L, Goldberg J, Petrascheck $M$, Ates G, Pinto-Duarte A, Shokhirev MN, Schubert D, Maher $P$. Elevating acetyl-CoA levels reduces aspects of brain aging. Elife. 2019; 8:e47866.

https://doi.org/10.7554/eLife.47866

PMID: $\underline{31742554}$

95. He B, Nohara K, Park N, Park YS, Guillory B, Zhao Z, Garcia JM, Koike N, Lee CC, Takahashi JS, Yoo SH, Chen Z. The Small Molecule Nobiletin Targets the Molecular Oscillator to Enhance Circadian Rhythms and Protect against Metabolic Syndrome. Cell Metab. 2016; 23:610-21.

https://doi.org/10.1016/j.cmet.2016.03.007 PMID:27076076

96. Shinozaki A, Misawa K, Ikeda Y, Haraguchi A, Kamagata M, Tahara Y, Shibata S. Potent Effects of Flavonoid Nobiletin on Amplitude, Period, and Phase of the Circadian Clock Rhythm in PER2::LUCIFERASE Mouse Embryonic Fibroblasts. PLoS One. 2017; 12:e0170904. https://doi.org/10.1371/journal.pone.0170904 PMID:28152057

97. Grabert K, Michoel T, Karavolos MH, Clohisey S, Baillie JK, Stevens MP, Freeman TC, Summers KM, McColl BW. Microglial brain region-dependent diversity and selective regional sensitivities to aging. Nat Neurosci. 2016; 19:504-16.

https://doi.org/10.1038/nn.4222

PMID:26780511

98. Galatro TF, Holtman IR, Lerario AM, Vainchtein ID, Brouwer N, Sola PR, Veras MM, Pereira TF, Leite REP, Möller T, Wes PD, Sogayar MC, Laman JD, et al. Transcriptomic analysis of purified human cortical microglia reveals age-associated changes. Nat Neurosci. 2017; 20:1162-71.

https://doi.org/10.1038/nn.4597

PMID:28671693

99. Ayata P, Badimon A, Strasburger HJ, Duff MK, Montgomery SE, Loh YE, Ebert A, Pimenova AA, Ramirez BR, Chan AT, Sullivan JM, Purushothaman I, Scarpa JR, et al. Epigenetic regulation of brain regionspecific microglia clearance activity. Nat Neurosci. 2018; 21:1049-60.

https://doi.org/10.1038/s41593-018-0192-3

PMID:30038282

100. Hammond TR, Dufort C, Dissing-Olesen L, Giera S, Young A, Wysoker A, Walker AJ, Gergits F, Segel M, Nemesh J, Marsh SE, Saunders A, Macosko E, et al. Single-Cell RNA Sequencing of Microglia throughout the Mouse Lifespan and in the Injured Brain Reveals Complex Cell-State Changes. Immunity. 2019; 50:253-71.e6. 
https://doi.org/10.1016/j.immuni.2018.11.004

PMID:30471926

101.Ito N, Sasaki K, Takemoto $H$, Kobayashi $Y$, Isoda $H$, Odaguchi $H$. Emotional Impairments and Neuroinflammation are Induced in Male Mice Invulnerable to Repeated Social Defeat Stress. Neuroscience. 2020; 443:148-63.

https://doi.org/10.1016/j.neuroscience.2020.07.023 PMID:32707290

102. Hori A, Ito N, Oikawa T, Hanawa T. Kososan, but not milnacipran, elicits antidepressant-like effects in a novel psychological stress-induced mouse model of depression. Trad \& Kampo Med. 2015; 2:1-7. https://doi.org/10.1002/tkm2.1013

103.Liu MY, Yin CY, Zhu LJ, Zhu XH, Xu C, Luo CX, Chen H, Zhu DY, Zhou QG. Sucrose preference test for measurement of stress-induced anhedonia in mice. Nat Protoc. 2018; 13:1686-98. https://doi.org/10.1038/s41596-018-0011-z PMID:29988104

104.Steru L, Chermat R, Thierry B, Simon P. The tail suspension test: a new method for screening antidepressants in mice. Psychopharmacology (Berl). 1985; 85:367-70.

https://doi.org/10.1007/BF00428203 PMID:3923523

105. Martin M. Cutadapt removes adapter sequences from high-throughput sequencing reads. EMBnet Bioinformatics in Action. 2011; 17:3. https://doi.org/10.14806/ej.17.1.200

106.Bolger AM, Lohse M, Usadel B. Trimmomatic: a flexible trimmer for Illumina sequence data. Bioinformatics. 2014; 30:2114-20. https://doi.org/10.1093/bioinformatics/btu170 PMID:24695404

107.Langmead B, Salzberg SL. Fast gapped-read alignment with Bowtie 2. Nat Methods. 2012; 9:357-9. https://doi.org/10.1038/nmeth.1923 PMID:22388286
108.Kim D, Paggi JM, Park C, Bennett C, Salzberg SL. Graph-based genome alignment and genotyping with HISAT2 and HISAT-genotype. Nat Biotechnol. 2019; 37:907-15. https://doi.org/10.1038/s41587-019-0201-4 PMID:31375807

109.Li H, Handsaker B, Wysoker A, Fennell T, Ruan J, Homer N, Marth G, Abecasis G, Durbin R, and 1000 Genome Project Data Processing Subgroup. The Sequence Alignment/Map format and SAMtools. Bioinformatics. 2009; 25:2078-9.

https://doi.org/10.1093/bioinformatics/btp352 PMID:19505943

110.Liao Y, Smyth GK, Shi W. featureCounts: an efficient general purpose program for assigning sequence reads to genomic features. Bioinformatics. 2014; 30:923-30.

https://doi.org/10.1093/bioinformatics/btt656

PMID:24227677

111.Robinson MD, Oshlack A. A scaling normalization method for differential expression analysis of RNAseq data. Genome Biol. 2010; 11:R25. https://doi.org/10.1186/gb-2010-11-3-r25 PMID:20196867

112.Robinson MD, McCarthy DJ, Smyth GK. edgeR: a Bioconductor package for differential expression analysis of digital gene expression data. Bioinformatics. 2010; 26:139-40. https://doi.org/10.1093/bioinformatics/btp616 PMID:19910308 


\section{SUPPLEMENTARY MATERIALS}

\section{Supplementary Figures}

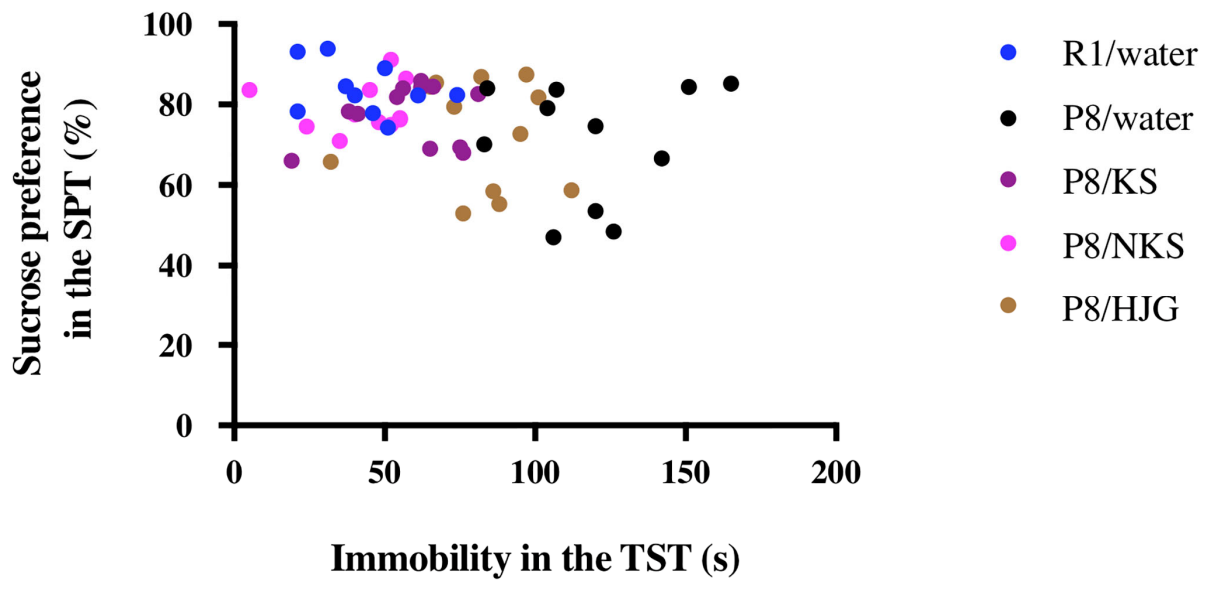

Supplementary Figure 1. Data in the TST were inversely correlated with those in the SPT. A single data point is shown for each mouse. Pearson's correlation coefficient test was performed $(r=-0.3048, n=57, P<0.05)$. 
A
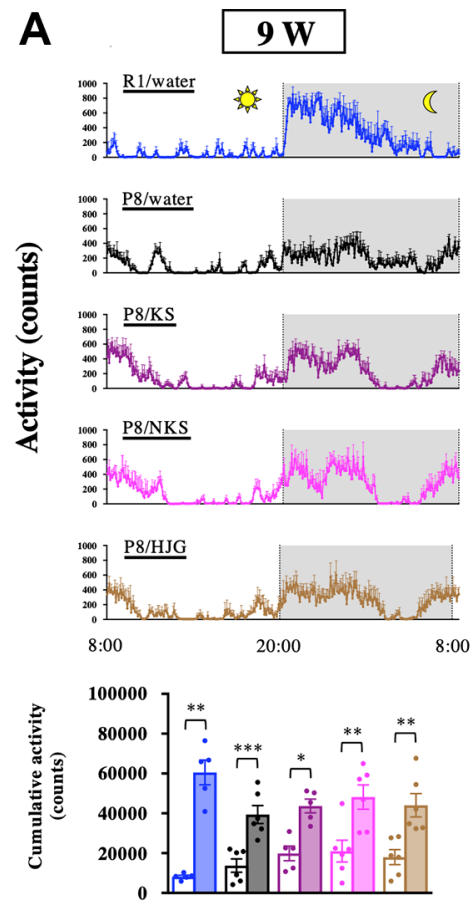

C
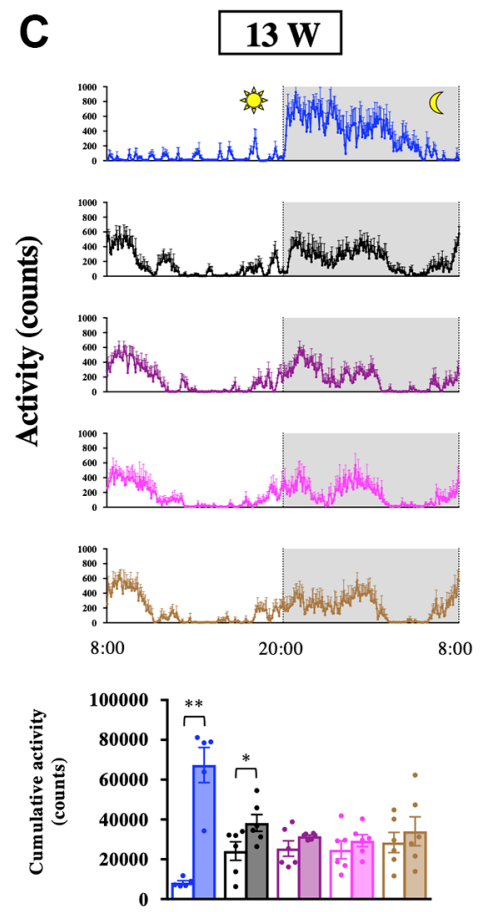

B

$11 \mathrm{~W}$
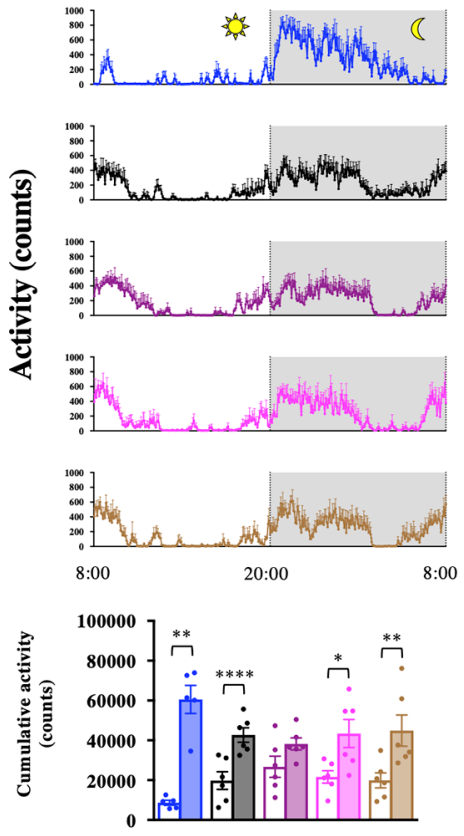

अ

$\square \square$ R1/water

$\square \square$ P8/water

$\square \square$ P8/KS

P8/NKS

P8/HJG

D

\section{$15 \mathrm{~W}$}
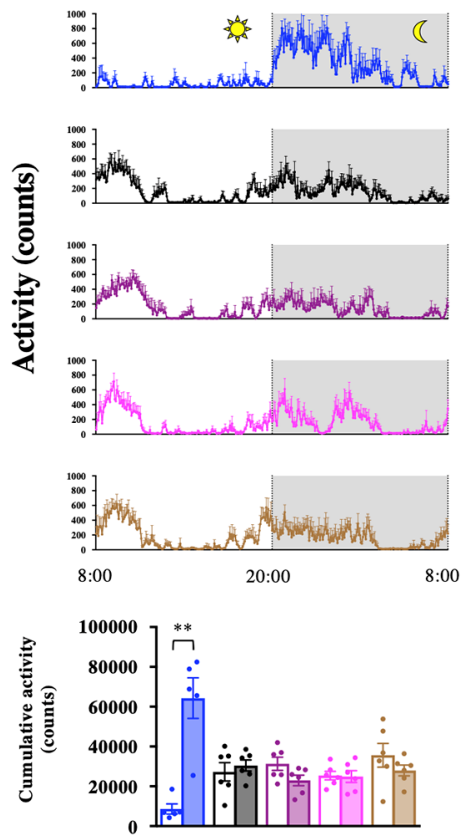

Supplementary Figure 2. The timeline of alterations in circadian rhythm in SAMP8 mice. Time course of locomotor activity of mice in their home cages and the cumulative activity during the diurnal and nocturnal phases are shown at 9 (A), 11 (B), 13 (C), and 15 (D) weeks of age. Data are shown as the mean $\pm \operatorname{SEM}(n=5$ or 6$) .{ }^{*} P<0.05,{ }^{* *} P<0.01,{ }^{* * *} P<0.001$, and ${ }^{* * * *} P<0.0001$, using paired $t$-test. 

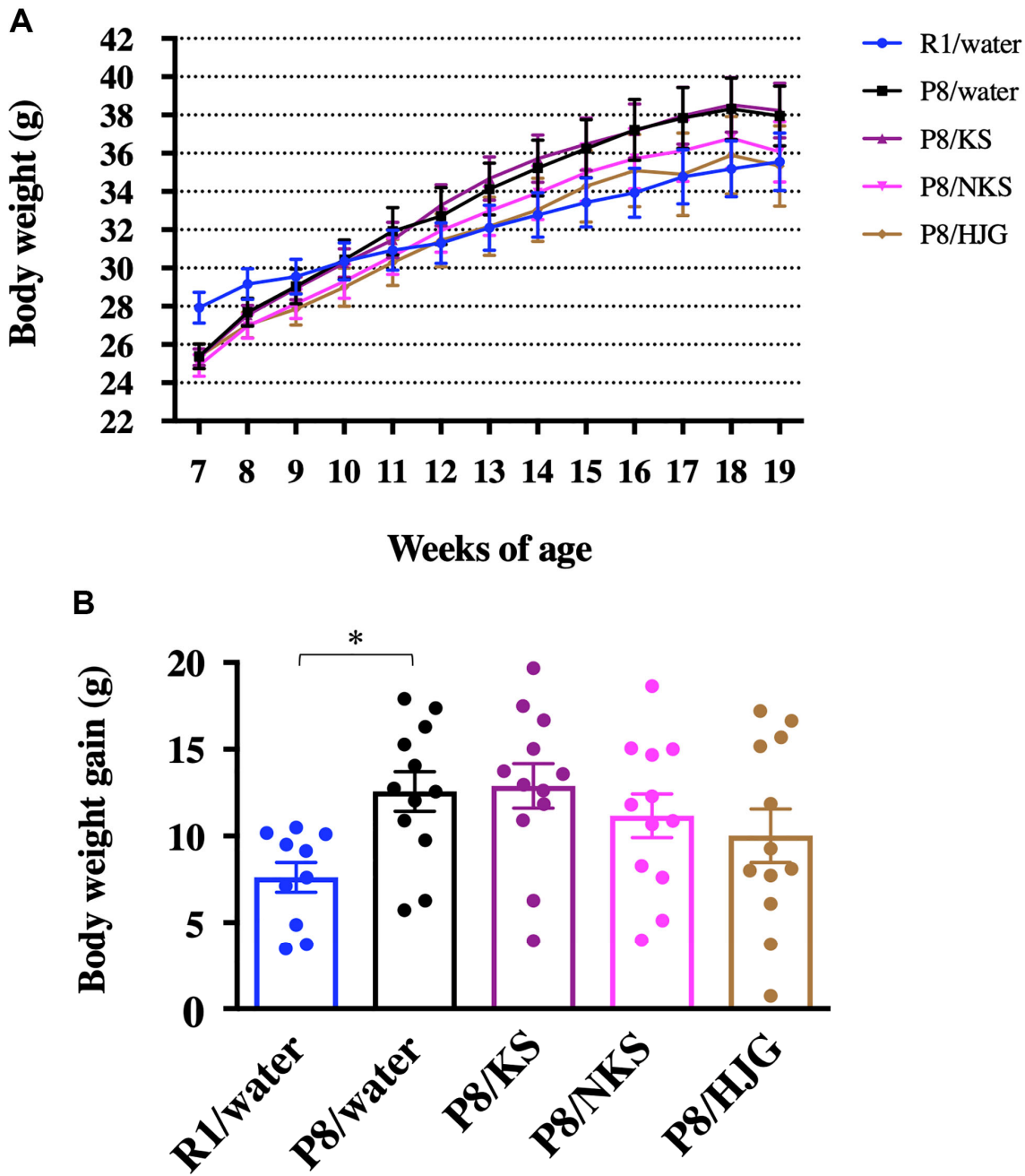

Supplementary Figure 3. Body weight gain is not affected by treatment with Kampo formulas. (A) Temporal changes in body weight. (B) Body weight gain of mice from 7 to 19 weeks of age. Data are shown as the mean \pm SEM $(n=10-12)$. ${ }^{*} P<0.05$ using Bonferroni's post-hoc test.

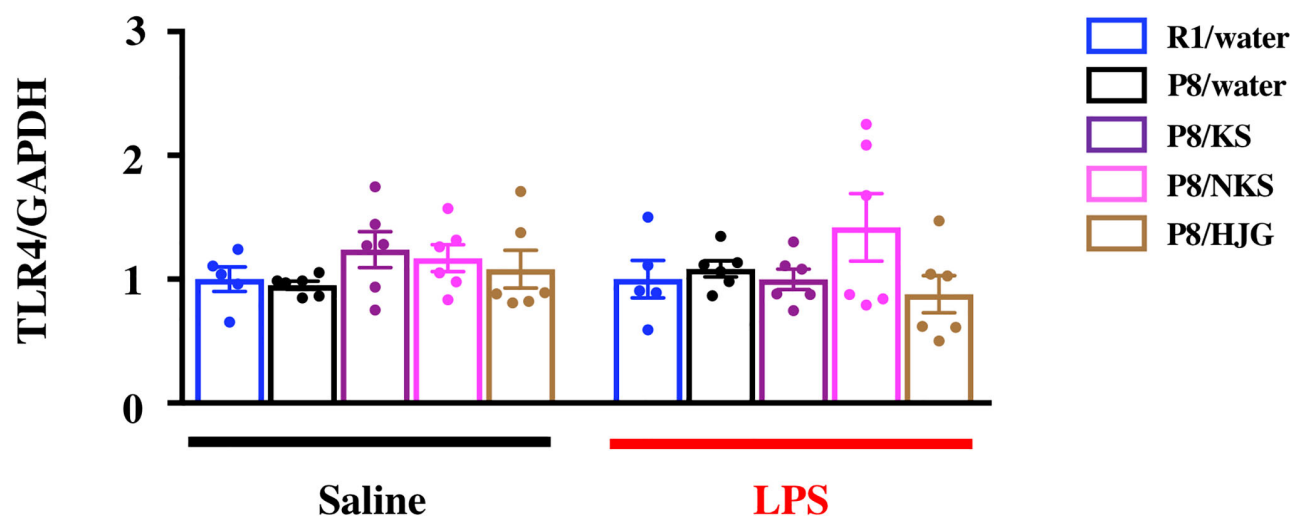

Supplementary Figure 4. TLR4 expression in the hippocampus of SAMP8 mice is not affected by oral administration of Kampo formulas. Expression of TLR4 was normalized based on GAPDH. Data are shown as the mean \pm SEM $(n=5$ or 6$)$. 


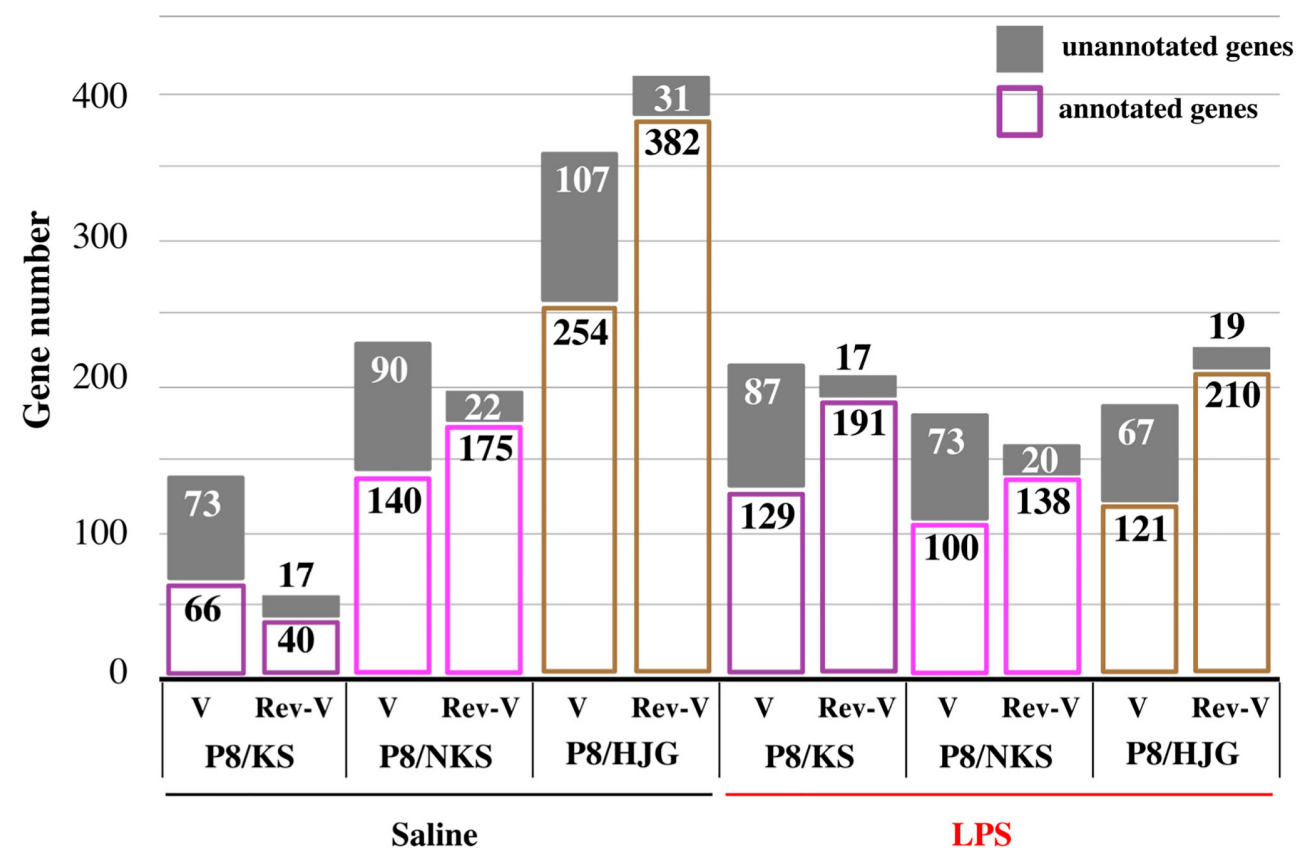

Supplementary Figure 5. Bar plot showing the distribution of the number of annotated and unannotated genes among the significantly recovered genes in the hippocampus of mice treated with Kampo formulas that were then administered saline or LPS. The number in each column represents the number of genes, which corresponds to the data in Figure 5B. 

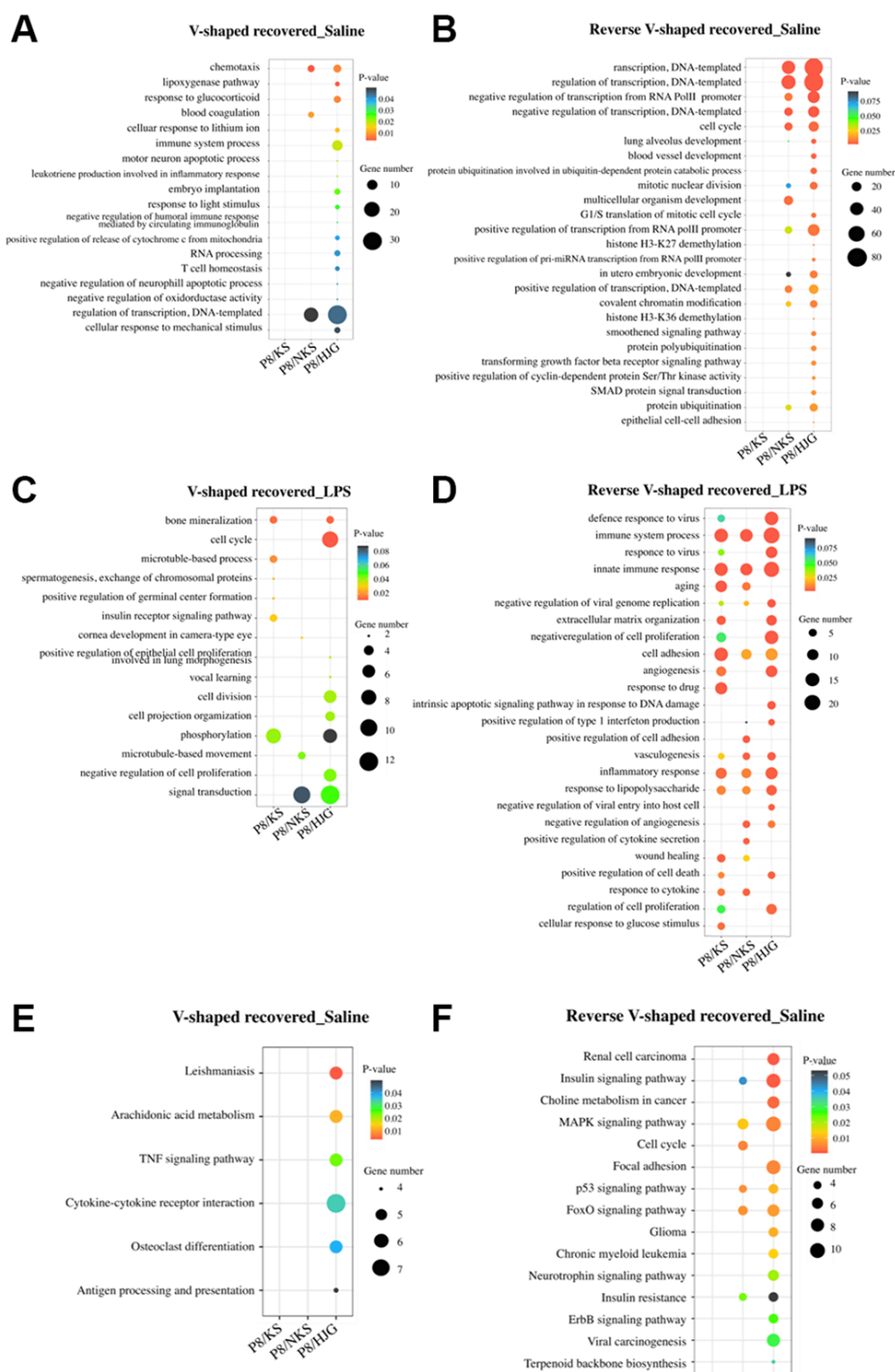

F Reverse V-shaped recovered_Saline
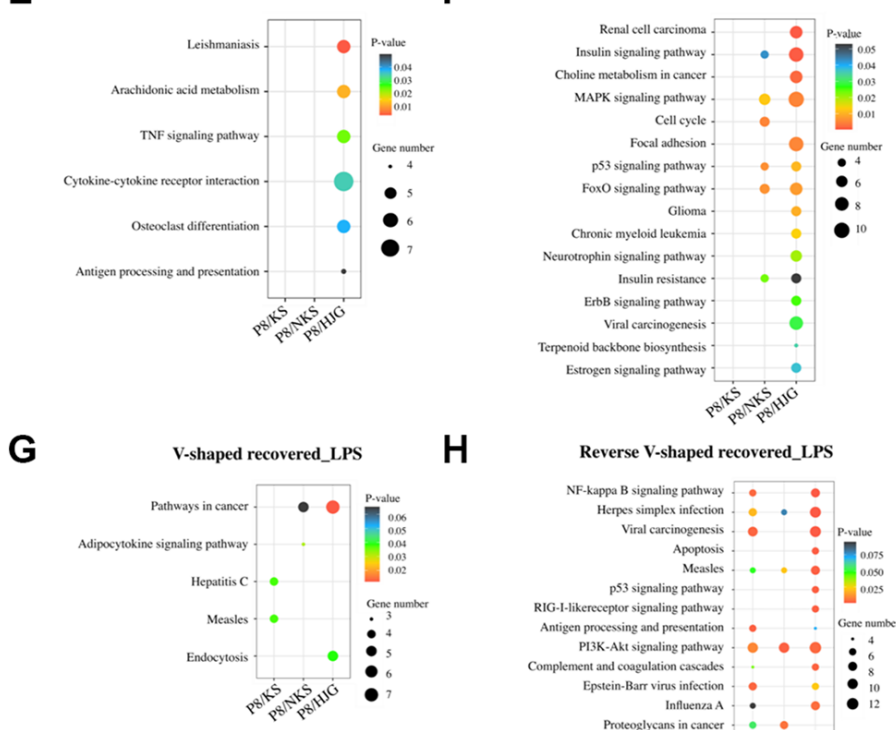

H

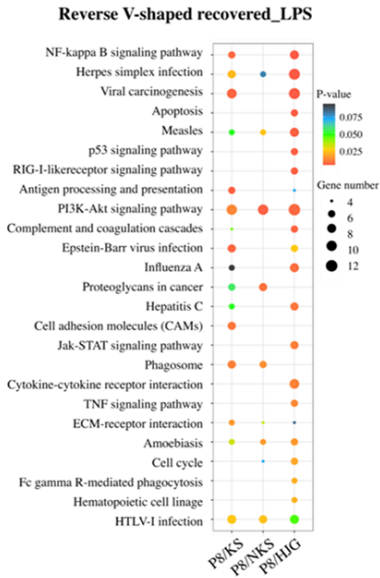

Supplementary Figure 6. Bubble plot showing GO analysis an dKEGG signaling pathway analysis of recovered DEGs in the hippocampus of SAMP8 mice in response to treatment with Kampo formulas. GO analysis of (A) all V-shaped recovered DEGs and (B) the top 25 reverse V-shaped recovered DEGs from saline-injected mice. GO analysis of (C) all V-shaped recovered DEGs and (D) the top 25 reverse V-shaped recovered DEGs from LPS- injected mice. KEGG signaling pathway enrichment analysis of (E) all V-shaped recovered and $(\mathbf{F})$ all reverse (V-shaped recovered DEGs with saline injection. KEGG signaling pathway enrichment analysis of (G) all V-shaped recovered DEGs and $(\mathbf{H})$ the top 25 reverse V-shaped recovered DEGs with LPS injection. 


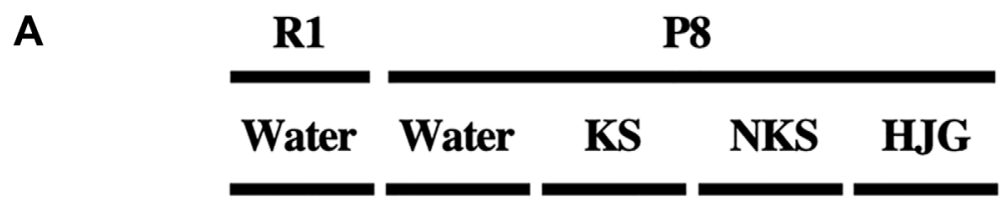

Tau

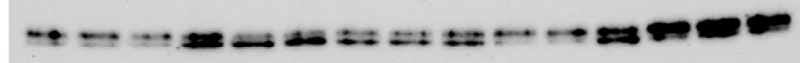

\section{GAPDH}

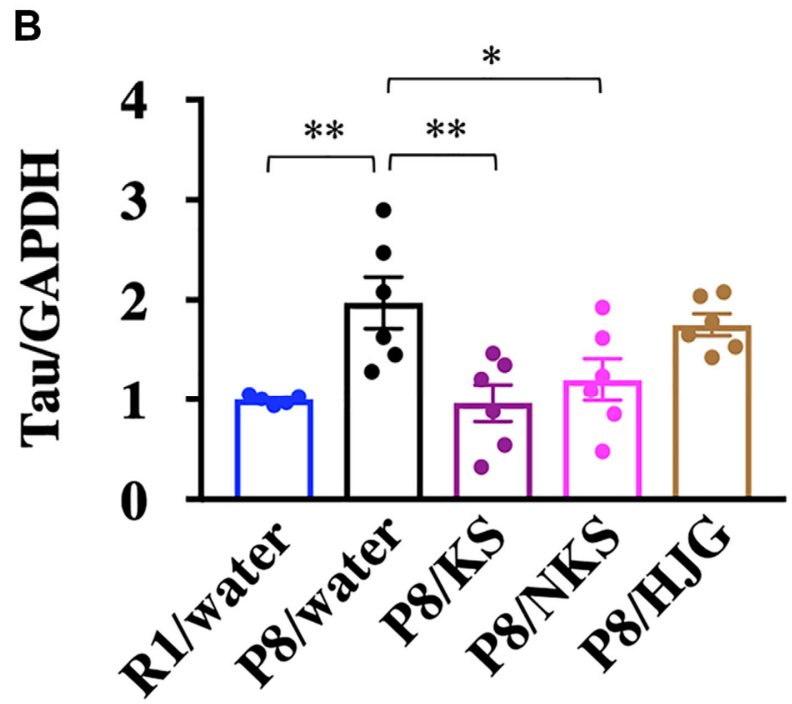

Supplementary Figure 7. Oral administration of KS and NKS, but not HJG, prevents the increase in tau in the brains of SAMP8 mice. (A) Representative western blotting images of tau and GAPDH expression. (B) Expression of tau was normalized based on GAPDH expression. Data are shown as the mean \pm SEM $(n=5$ or 6$) .{ }^{*} P<0.05$ and ${ }^{* *} P<0.01$ using Bonferroni's post-hoc test. 

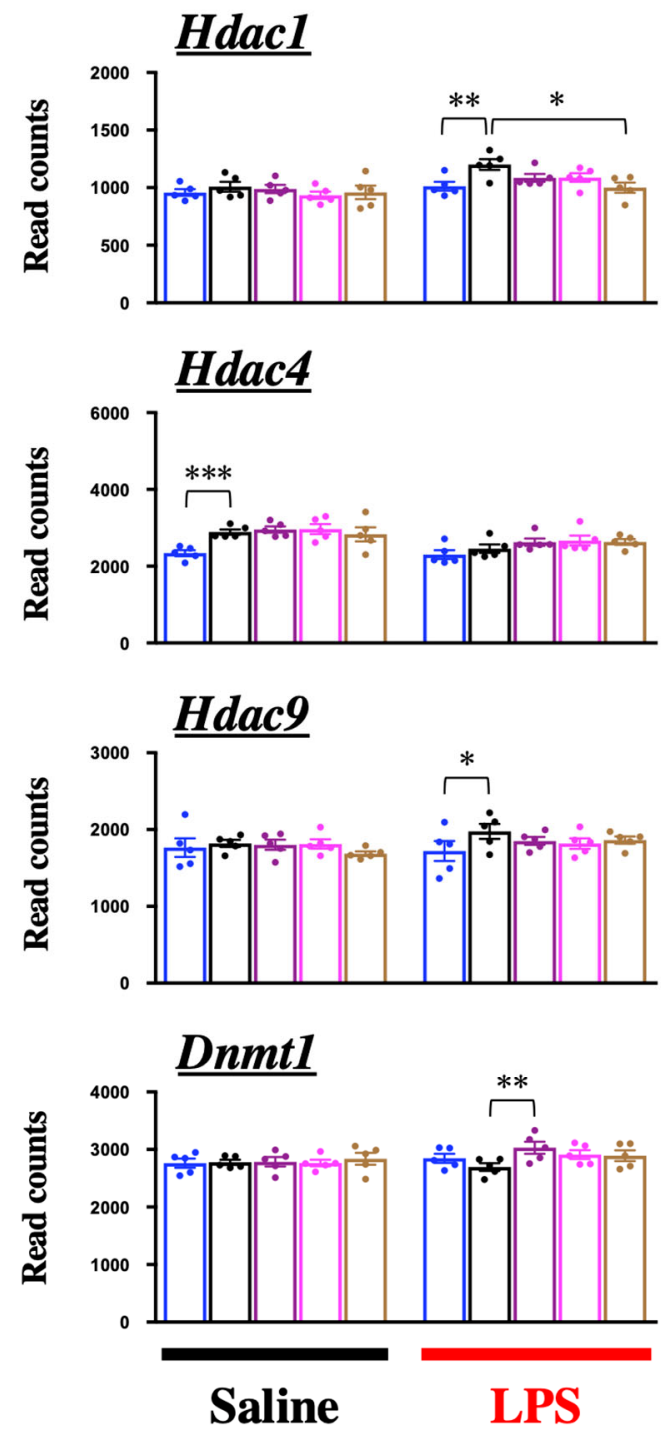
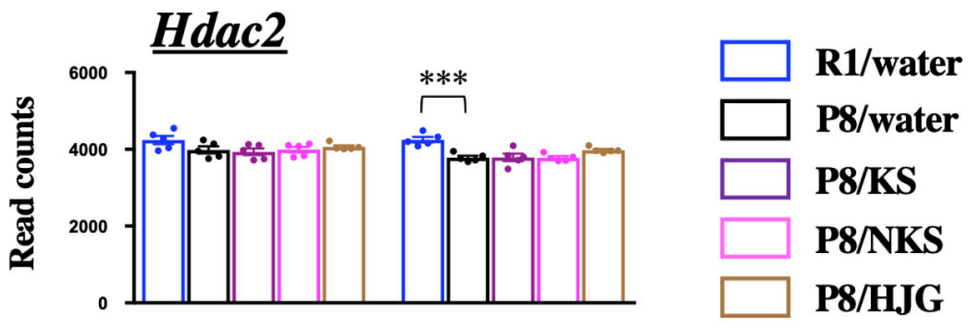

\section{$\underline{\text { Hdac5 }}$}

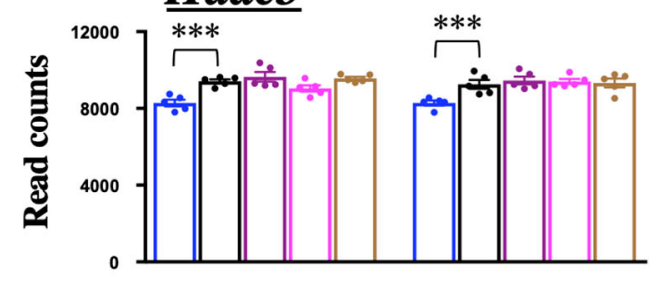

\section{$\underline{\text { Hdac10 }}$}
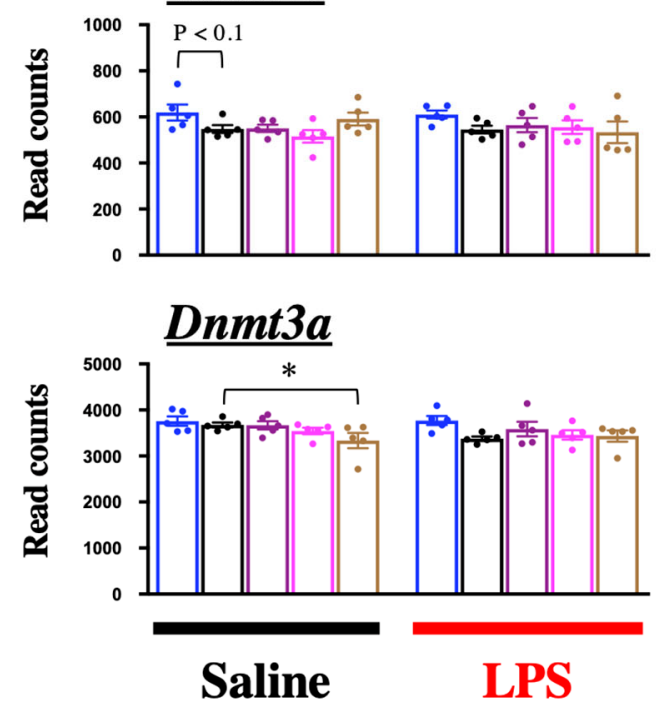

Supplementary Figure 8. Hippocampal gene expression of epigenetic-related genes in Kampo formula-administered mice after LPS or saline injection. Data are shown as the mean $\pm \operatorname{SEM}(n=5) .{ }^{*} P<0.05,{ }^{* *} P<0.01$, and ${ }^{* * *} P<0.001$ using Bonferroni's posthoc test. 


\section{Supplementary Tables}

Please browse Full Text version to see the data of Supplementary Table 1.

Supplementary Table 1. A full list of significantly V-shaped recovered genes under physiological condition.

Supplementary Table 2. Amount of the three polymethoxyflavonces-nobiletin, sinensetin, and tangeretin-in 1-gram water extracts of kososan (KS) and nobiletin-rich kososan (NKS).

\begin{tabular}{lccc}
\hline & Nobiletin & Sinensetin & Tangeretin \\
\hline KS & $0.09 \mathrm{mg}$ & N.D. & $0.02 \mathrm{mg}$ \\
NKS & $2.44 \mathrm{mg}$ & $0.142 \mathrm{mg}$ & $0.516 \mathrm{mg}$ \\
\hline
\end{tabular}

For this analysis, water extracts of KS and NKS was analyzed by high performance liquid chromatography under the following conditions: For determination of nobiletin, sinensetin, and tangeretin contents, an ODS column (Mightysil RP-18 GP S, $4.6 \times$ $150 \mathrm{~mm}, 5 \mu \mathrm{m}$; Kanto Chemical Co., INC., Japan), equipped with a detector (an ultraviolet absorption photometer, wavelength: $338 \mathrm{~nm}$ ) and operated at a column temperature of $40^{\circ} \mathrm{C}$, mobile phase of $40 \%$ acetonitrile in $\mathrm{H}_{2} \mathrm{O}$, and a flow rate of $0.8 \mathrm{ml} / \mathrm{min}$ was used. An injection volume of $10 \mu \mathrm{L}$ was used for all standard solutions and sample solutions tested. Abbreviation: N.D.: not detected. 


\section{Supplementary Table 3. Unannotated genes that showed significant recovery after Kampo formula treatment and a significant positive or negative correlation with immobility in the TST.}

\begin{tabular}{|c|c|c|c|c|c|c|c|c|c|c|c|c|}
\hline \multirow{2}{*}{ Gene_ID } & \multirow[b]{2}{*}{ Gene_name } & \multicolumn{5}{|c|}{ Average read count $(n=5)$} & \multicolumn{4}{|c|}{$P$-value (vs. P8/water) } & \multicolumn{2}{|c|}{ Correlation (vs. TST) } \\
\hline & & R1/water & P8/water & $\mathbf{P 8} / \mathrm{KS}$ & P8/NKS & P8/HJG & R1/water & $\mathbf{P 8} / \mathrm{KS}$ & P8/NKS & P8/HJG & $\mathbf{r}$ & $P$-value \\
\hline \multicolumn{13}{|c|}{ Saline/V-shaped recovered genes } \\
\hline ENSMUSG00000082964 & Rpl13-ps5 & 3.21 & 0.20 & 4.01 & 4.45 & 1.20 & 0.002 & 0.001 & 0.000 & 0.086 & -0.419 & 0.0369 \\
\hline ENSMUSG00000073752 & Gm10570 & 24.84 & 9.82 & 18.44 & 18.56 & 13.34 & 0.001 & 0.032 & 0.030 & 0.310 & -0.592 & 0.0018 \\
\hline ENSMUSG00000077637 & Gm22771 & 138.37 & 105.13 & 122.84 & 131.52 & 117.13 & 0.010 & 0.149 & 0.037 & 0.320 & -0.410 & 0.0419 \\
\hline ENSMUSG00000081431 & Gm15483 & 22.83 & 13.29 & 21.87 & 22.55 & 21.38 & 0.040 & 0.060 & 0.045 & 0.073 & -0.581 & 0.0023 \\
\hline ENSMUSG00000102863 & Gm37639 & 12.02 & 3.22 & 7.22 & 9.87 & 5.47 & 0.002 & 0.060 & 0.008 & 0.231 & -0.590 & 0.0019 \\
\hline ENSMUSG00000052865 & Gm13619 & 7.21 & 2.01 & 6.63 & 8.08 & 3.84 & 0.018 & 0.028 & 0.009 & 0.250 & -0.577 & 0.0025 \\
\hline ENSMUSG00000097430 & Gm10544 & 22.64 & 7.84 & 16.04 & 17.74 & 8.49 & 0.000 & 0.006 & 0.001 & 0.779 & -0.441 & 0.0274 \\
\hline ENSMUSG00000097648 & 9330185C12Rik & 9.61 & 3.03 & 5.21 & 10.27 & 4.65 & 0.020 & 0.289 & 0.014 & 0.405 & -0.419 & 0.0371 \\
\hline ENSMUSG00000100455 & $\mathrm{Gm} 29170$ & 398.86 & 334.44 & 380.46 & 394.50 & 364.66 & 0.013 & 0.070 & 0.020 & 0.226 & -0.612 & 0.0012 \\
\hline ENSMUSG00000106565 & Gm43582 & 14.83 & 4.23 & 7.02 & 12.71 & 6.46 & 0.001 & 0.195 & 0.003 & 0.284 & -0.455 & 0.0223 \\
\hline ENSMUSG00000103283 & $\begin{array}{c}\text { ENSMUSG0000 } \\
0103283\end{array}$ & 87.70 & 68.78 & 82.17 & 91.76 & 75.62 & 0.042 & 0.139 & 0.016 & 0.434 & -0.503 & 0.0103 \\
\hline ENSMUSG00000056418 & BC043934 & 33.25 & 16.43 & 27.45 & 27.86 & 14.98 & 0.005 & 0.045 & 0.039 & 0.731 & -0.415 & 0.0392 \\
\hline \multicolumn{13}{|c|}{ Saline/reverse V-shaped recovered genes } \\
\hline ENSMUSG00000105003 & Gm40055 & 3.21 & 9.64 & 6.02 & 3.63 & 6.88 & 0.019 & 0.281 & 0.034 & 0.436 & 0.552 & 0.0043 \\
\hline ENSMUSG00000083914 & Rps18-ps1 & 0.40 & 14.04 & 9.22 & 6.47 & 12.08 & 0.000 & 0.139 & 0.009 & 0.585 & 0.525 & 0.0071 \\
\hline ENSMUSG00000047509 & Gm6776 & 7.62 & 16.33 & 9.83 & 6.86 & 12.09 & 0.036 & 0.154 & 0.018 & 0.390 & 0.476 & 0.0162 \\
\hline \multicolumn{13}{|c|}{ LPS/V-shaped recovered genes } \\
\hline ENSMUSG00000100636 & Gm3551 & 5.55 & 0.40 & 3.60 & 4.79 & 1.99 & 0.002 & 0.013 & 0.004 & 0.080 & -0.591 & 0.0019 \\
\hline ENSMUSG00000065694 & $\mathrm{Gm} 25411$ & 6.97 & 1.78 & 4.61 & 5.99 & 3.58 & 0.011 & 0.084 & 0.024 & 0.215 & -0.413 & 0.0400 \\
\hline ENSMUSG00000097330 & $\mathrm{Gm} 26672$ & 17.08 & 7.91 & 10.97 & 15.56 & 10.93 & 0.019 & 0.334 & 0.041 & 0.340 & -0.477 & 0.0159 \\
\hline ENSMUSG00000073371 & Gm6594 & 34.74 & 21.75 & 33.51 & 33.68 & 33.02 & 0.030 & 0.046 & 0.043 & 0.054 & -0.399 & 0.0482 \\
\hline ENSMUSG00000087063 & Gm15857 & 8.96 & 2.39 & 6.79 & 9.37 & 6.77 & 0.003 & 0.020 & 0.002 & 0.020 & -0.413 & 0.0401 \\
\hline ENSMUSG00000098164 & Gm5493 & 9.18 & 2.97 & 5.19 & 9.17 & 3.59 & 0.032 & 0.303 & 0.032 & 0.733 & -0.530 & 0.0065 \\
\hline ENSMUSG00000069011 & Gm10254 & 117.32 & 76.23 & 99.04 & 132.70 & 88.94 & 0.039 & 0.211 & 0.008 & 0.462 & -0.472 & 0.0171 \\
\hline ENSMUSG00000084828 & Gm12367 & 17.11 & 9.28 & 13.17 & 16.56 & 11.33 & 0.030 & 0.224 & 0.040 & 0.494 & -0.546 & 0.0048 \\
\hline ENSMUSG00000102728 & Gm37934 & 3.98 & 0.00 & 1.60 & 2.19 & 0.99 & 0.000 & 0.008 & 0.003 & 0.024 & -0.465 & 0.0191 \\
\hline ENSMUSG00000044081 & Zfp85os & 87.82 & 48.61 & 65.45 & 71.40 & 59.12 & 0.000 & 0.073 & 0.020 & 0.240 & -0.594 & 0.0017 \\
\hline ENSMUSG00000102193 & Gm7299 & 5.57 & 0.40 & 3.39 & 5.80 & 2.98 & 0.015 & 0.049 & 0.014 & 0.065 & -0.565 & 0.0033 \\
\hline \multicolumn{13}{|c|}{ LPS/reverse V-shaped recovered genes } \\
\hline ENSMUSG00000097301 & AW121686 & 54.35 & 74.35 & 50.89 & 45.87 & 51.94 & 0.032 & 0.010 & 0.001 & 0.015 & 0.558 & 0.0037 \\
\hline ENSMUSG00000085896 & 5330429C05Rik & 7.37 & 20.00 & 14.19 & 7.17 & 15.70 & 0.034 & 0.449 & 0.030 & 0.590 & 0.490 & 0.0130 \\
\hline ENSMUSG00000077316 & n-R5s171 & 0.20 & 4.36 & 3.40 & 1.20 & 5.98 & 0.000 & 0.651 & 0.040 & 0.551 & 0.590 & 0.0019 \\
\hline ENSMUSG00000080773 & Gm12955 & 1.19 & 6.94 & 2.99 & 2.00 & 3.18 & 0.007 & 0.153 & 0.043 & 0.183 & 0.554 & 0.0040 \\
\hline ENSMUSG00000086326 & Gm13200 & 6.18 & 13.63 & 9.98 & 4.99 & 12.13 & 0.022 & 0.342 & 0.005 & 0.719 & 0.651 & 0.0004 \\
\hline
\end{tabular}

Correlation analysis was performed using Pearson's correlation coefficient. 